Noname manuscript No.

(will be inserted by the editor)

\title{
Diagnosis and Prognosis of Mental Disorders by means of EEG and Deep Learning: A Systematic Mapping Study
}

\author{
Manuel J. Rivera • Miguel A. Teruel • \\ Alejandro Maté • Juan Trujillo
}

Received: date / Accepted: date

\begin{abstract}
Electroencephalography (EEG) is used in the diagnosis and prognosis of mental disorders because it provides brain biomarkers. However, only highly trained doctors can interpret EEG signals due to its complexity. Machine learning has been successfully trained with EEG signals for classifying mental disorders, but a time consuming and disorder-dependant feature engineering (FE) and subsampling process is required over raw EEG data. Deep Learning (DL) is positioned as a prominent research field to process EEG data because (i) it features automated FE by taking advantage of raw EEG signals improving results and (ii) it can be trained over the vast amount of data generated by EEG. In this work, a systematic mapping study has been performed with 46 carefully selected primary studies. Our goals were (i) to provide a clear view of which are the most prominent study topics in diagnosis and prognosis of mental disorders by using EEG with DL, and (ii) to give some recommendations for future works. Some results are: epilepsy was the predominant mental disorder present in around half of the studies, convolutional neural networks also appear in approximate $50 \%$ of the works. The main conclusions are (i) processing EEG with DL to detect mental disorders is a promising research field and (ii) to objectively compare performance between studies: public datasets, intra-subject validation, and standard metrics should be used. Additionally, we suggest to pay more attention to ease the reproducibility, and to use (when possible) an available framework to explain the results of the created DL models.
\end{abstract}

Keywords Deep Learning · Diagnosis · Electroencephalogram · Systematic Mapping Study · Mental Disorder · Prognosis

F. Author

first address

Tel.: +123-45-678910

Fax: +123-45-678910

E-mail: fauthor@example.com

S. Author

second address 


\section{Introduction}

There are more than 150 recognised mental disorders (Association et al. 2013), some of them having high prevalence in the population. Indeed, it is estimated that $38.2 \%$ of the European population suffer some mental disorder with a 12month prevalence, that is 164.8 million people (Wittchen et al. 2011). The four most common mental disorders are anxiety, insomnia, major depression and somatoform (diseases characterized by various discomforts that afflict the patient but which cannot be explained by the existence of an organic lesion). Therefore, an early and accurate diagnostic of mental disorders could improve the quality of life of patients. Regarding such diagnosis, one of the most commonly used techniques is Electroencephalogram (EEG), which provides information from patient's brain that can aid to develop a trustworthy diagnostic.

EEG is performed by using a noninvasive device that captures the electrical brain activity produced by its upper layers. It consists of an array of electrodes which are placed over the scalp of the patient. EEG is widely used in the diagnosis and prognosis of mental disorders, such as epilepsy, which is currently a very active research field ( $\mathrm{Li}$ et al. 2019b; Sharma and Pachori 2017; Zhang and Chen 2016; Sharma et al. 2018). EEG provides objective biomarkers from the patient's brain (Olbrich and Arns 2013; Jeste, Frohlich, and Loo 2015; Olbrich, Van Dinteren, and Arns 2016; Mcloughlin, Makeig, and Tsuang 2014). These biomarkers can be recorded because of the high temporal resolution of EEG, which for some devices can reach up to $5000 \mathrm{~Hz}$ (see Figure 9). This high-sample frequency provides a continuous lecture of the brain function. Besides, other techniques such as Magnetic Resonance Imaging (MRI) and Positron Emission Tomography (PET), although provide a full spatial brain map, only capture a static brain image. Furthermore, EEG is a relatively cheap technology since devices cost from in 700 Euros for Emotiv wearable devices and rise up to 20000 Euros in the case of a g.tec system (Zerafa et al. 2018). EEG also enables ambulatory diagnostic, thus reducing the costs of the health system (Dash et al. 2012; Faulkner, Arima, and Mohamed 2012).

Traditionally, the interpretation of the EEG records is performed by doctors who have been trained for hundred of hours. Manually analyzing EEG records is a time-consuming task since the length of the recorded data ranges may vary from hours to days. In order to try to automate the process, Artificial Intelligence (AI) techniques have been proposed to efficiently analyzing these signals, thus minimizing human intervention. The more typical AI task is a classification, where an algorithm is trained to distinguish whether a patient has a mental disorder or not. The main advantages of using AI are (i) to provide a more objective diagnosis and prognosis avoiding the cognitive bias (Croskerry 2003) because AI over EEG only use biometric signals and (ii) to enable us to join EEG signals with other biometrics data captured from the patient such as Electrocardiogram (ECG) and Eye Movement(EM), among others, trying to increase the detection accuracy. Machine learning (ML) is a subclass of AI techniques which has been proposed to automatize the processing of these signals (Podgorelec 2012; Acharya et al. 2012). However, a highly manual and time-consuming Feature Engineering (FE) process, including channel selection (Baig, Aslam, and Shum 2020), is still needed for tra- 
ditional ML algorithms to achieve reasonable accuracy levels. To construct the input features for a ML algorithm, it is necessary to manually compound features that make explicit the information that is contained in the high-complexity raw EEG data (Sun and Zhou 2014). Moreover, the FE stage is task-dependent, so this hand-crafted process needs to be adjusted to each mental illness.

Deep Learning (DL) is, in turn, subset of ML algorithms that extend the concept of the Neural Networks (NNs) by incorporating more than one hidden layer. DL has successfully been used with a wide range of applications such as computer vision, natural language processing and signal processing. A key feature of DL algorithms is that using their hidden layers, they develop an automatic FE, saving considerable human time and effort although at the cost of the capability to explain the FE carried out. DL is positioning as an alternative to process EEG data because this automation of the FE stage minimizes human intervention. It also can improve the classification accuracy since DL can deal with the raw EEG data by avoiding the information loss produced in an hand-engineered FE. Because of the previously stated reasons, we are only focusing on DL techniques. The number of studies where DL is used to process EEG data to diagnose or prognose a mental disorder has been increasing over the time (see Figure 12). This fact indicates that it is an open and promising research field. We consider that a wide number of studies can be performed in this field since (i) the high number of mental disorders, DL can process EEG biomarkers to try to automate a more objective diagnosis or prognosis, (ii) DL is an AI field in constant evolution where continuously new algorithms are presented which outperform the previous ones. Therefore, when a new algorithm is released, a new research opportunity is open.

Some previous secondary studies regarding EEG and DL have already been performed (Merlin Praveena, Angelin Sarah, and Thomas George 2020; Craik, He, and Contreras-Vidal 2019; Roy et al. 2019). However, to the best of our knowledge, and as we will explain throughout the whole paper, there is still a need for continuing with this research topic (i) by focusing in the diagnosis or prognosis of mental disorders because of the high number of people that presents this type of disorders and (ii) to provide a clear mapping between mental disorders and DL algorithms for detecting the main research areas and research gaps. These are the main reasons why, in this paper, we carry out a Systematic Mapping Study (SMS) for providing which mental disorders and DL techniques have been used for diagnosing and prognosing by means of EEG and DL. Therefore, we are discarding those works not forcing on diagnosis or prognosis. Additionally, in this scientific secondary study we present a mapping of mental disorders with DL techniques and additional biomarkers used. In this mapping, we expose both the main research areas and the existing research gaps in diagnosis and prognosis of mental disorders by means of EEG and DL. In other words, what is already done and what could be done is this field. To carry out this task, the selected primary works were mapped by using previously defined categories according to the research questions proposed in Section 4.1. Therefore, to the best of our knowledge, this is the first detailed systematic review work that analyzes and classifies all the current proposals that consider applying Deep Learning techniques on EEG for the diagnosis and prognosis of different mental disorders. 
The main purpose of this work is to provide a clear view of which are the most prominent study topics in diagnosis and prognosis of mental disorders by using EEG with DL, as well as to expose some issues we found. For each issue, we provide insights and directions on how to potentially address them. In this manner, we aim to contribute to make genuine progress in the diagnosis and prognosis of mental disorders by using EEG and DL. We strongly believe that this work will be a useful starting point to save time in finding which are the most adequate DL techniques and their best results in the diagnosis and prognosis of mental disorders. Moreover, offering a clear view of the existing issues and potential lines of research will help to foster new ideas and solutions.

The organization of the paper is as follows: in Section 2 EEG and DL concepts are briefly presented. Related works and motivation of this work can be found in Section 3. The methodology we followed to carry out this study, the research questions, databases used to retrieve papers, screening of studies, the keywording extraction and the mapping results are shown in Section 4. In Section 5 a discussion of the main insights is presented. Finally, in Section 6, shows the conclusions obtained from the study.

\section{EEG and Deep Learning}

In the following, EEG and Deep Learning (DL) will be introduced for the sake of the understandably of the rest of this manuscript. Hence, Section 2.1 will present EGG and after that, Section 2.2 will do so with regard to DL.

\subsection{EEG}

EEG devices consist of an array of electrodes which are placed in the scalp to capture the electrical signals produced not only by a single neuron but also in a brain region. These signals can be used as biomarkers to diagnose and prognose a wide range of mental illnesses. With regards to the position of the electrodes, the international 10-20 system is the most used one (Jurcak, Tsuzuki, and Dan 2007) which typically consists in 19 electrodes with 2 additional ones located near the ears (Mecarelli 2019). Moreover, high-density electrode systems such as 10-10 system with 81 channels and the 10-5 system with up to 345 electrodes are available. The 10-5 system has not yet been accepted by the American Clinical Neurophysiology Society or by the International Federation of Clinical Neurophysiology (Mecarelli 2019). Using an internationally recognized system fosters the study reproducibility.

EEG provides a high temporal resolution because of the high sample rate frequency that can be used in the data acquisition. For analyzing the EEG captured data, it is usually split into five frequency bands, namely, delta $<4 \mathrm{~Hz}$, theta 4 $8 \mathrm{~Hz}$, alpha $8-13 \mathrm{~Hz}$, beta 13-30 Hz and gamma >30 Hz (Hu and Zhang 2019). Some advantages that EEG provides over MRI and PET are the low cost price, the non-invasive nature and the possibility of performing an ambulatory diagnostic. This enables the study of the brain activity continuously, over hours or even 
days. In contrast, because of the sensibility of the EEG system, patient's muscle movements, eye blinks and heartbeats as well as the 50 or $60 \mathrm{~Hz}$ of the power lines are recorded too, thus producing interference. Hence, the signal needs to be cleaned of noise in a pre-processing stage, prior to their analysis.

\subsection{Deep Learning}

Neural Networks (NNs) are a type of ML algorithm which is composed by layers where artificial neurons or units are placed. NNs have three kind of layers called input, hidden and output. In the input layer, the input data is placed. Next, the hidden layer takes the input data and then a computation is done to end up transferring the result to the output layer where the final result is returned by the network. Neurons between layers are connected, thus having each of these connections a different weight. Neurons from the hidden and the output layers take their inputs and weights and carry out a dot product which result is passed to an activation function. Usually, this activation function is nonlinear. The inference in NNs is done by a process called Forward-Propagation where the information flows from the input to the output layers. For training the NNs, that is, adjusting the parameters or weights of each unit to produce the expected output, the Back-Propagation algorithm is used (Rumelhart, Hinton, and Williams 1986). In this algorithm, the calculated error in the output layer is "propagated" through the networks to adjust the weights of the connections. The amount added or subtracted for each weight is calculated with the gradient descent algorithm which use the direction obtained with the partial derivative of the nonlinear activation function over each weight. In this process a nonlinear boundary decision is "learned".

Deep Learning (DL) involves all NNs architectures which employ a more than one hidden layer. There are several DL architectures although the most commons are: Multilayer Perceptron (MLP), convolutional neural networks (CNN), Recurrent Neural Networks (RNN), Autoencoders (AE), and generative models (Shrestha and Mahmood 2019). A more detailed description of all these kinds of DL architectures is provided in Section 4.4.

In recent years DL has been widely applied to computer vision, natural language processing and signal processing tasks, achieving groundbreaking results in these fields. In healthcare, DL has become a relevant AI technique (Miotto et al. 2017; Domingues et al. 2019; Murtaza et al. 2019). Also, in recent years, DL has become a promising research topic to process EEG records (see Figure 12). The main advantage of processing EEG data with DL, is that, in contrast with traditional ML techniques, it can treat raw EEG data because it performs an automatic feature engineering (FE) or feature extraction. An automatic FE can extract new information from the raw data which can improve the classification result where performing hand-crafted FE is not feasible. In this sense, CNNs can process raw EEG data benefiting from spatial information, by applying convolutions over the input EEG data in various manner: $1 \mathrm{D}$ convolutions to process channels in a isolated form or $2 \mathrm{D}$ and $3 \mathrm{D}$ convolutions to process neighboring channels together (Wei et al. 2018; Phang et al. 2020; Mumtaz and Qayyum 2019). Raw EEG data, as sequence data, can also be processed by exploiting its temporal component. 
RNNs can benefit from this information by using feedback connections capable of learning from this sequence data (Warrick, Lostanlen, and Nabhan Homsi 2019). Machine learning techniques such as Decision Trees, KNN, or SVM are not capable of use raw EEG data due to (i) high dimensionality: e.g. an EEG that involves 19 channels with a sample rate of $256 \mathrm{~Hz}$ generates $292 \mathrm{k}$ records per minute, that is, $292 \mathrm{k}$ raw input features. As a result, this becomes a problem for ML algorithms because (i) the data volume translates into significant processing time, (ii) can translate into an overfitting problem, and (iii) they can not take advantage of the spatio-temporal information because there is not possible to inform the algorithm that a set of features are spatially together or form a sequence. Even when a huge amount of data is not available, DL is still a feasible method by using pre-trained networks. This technique is called transfer learning (Yosinski et al. 2014). For more details see Section 5 .

\section{Related works}

Some previous secondary studies has been done by reviewing the application of the AI field to process EEG data. Concerning to the use of ML, (Hosseini, Hosseini, and Ahi 2020) reviewed ML methods made for EEG analysis with bioengineering applications. They presented that all main ML algorithms that have been used for EEG classification tasks, such as emotion recognition, measure mental workload, sleep scoring and mental disorder among others. As stated in the introduction section, Feature Engineering is an important step prior to using ML over EEG data, (Noor and Ibrahim 2020) reviewed which EEG-extracted features are more useful for a favourable or unfavorable outcome prediction made by ML algorithms.

Regarding reviews in EEG with DL, (Merlin Praveena, Angelin Sarah, and Thomas George 2020) has focused in which DL techniques were used for EEG signal applications; (Craik, He, and Contreras-Vidal 2019) analysed which DL algorithms have been used for EEG classification tasks. Additionally, they (i) showed how the input data is presented to the algorithm: images, calculated features and signal values, and (ii) explore the existence of specifics DL algorithms for a specific type of task. Finally in (Roy et al. 2019) a systematic review is done for DL-based EEG analysis where they discuss the studies from various aspects: data, preprocessing methodology, DL design choices, results and reproducibility of the experiments.

To the best of our knowledge, this research is the first Systematic Mapping Study (SMS) done in diagnosis and prognosis of mental disorders by means of EEG and DL. We have analysed four main aspects in the selected studies: (i) which mental disorders have been diagnosed and prognosed, (ii) DL techniques used, (iii) additional used biomarkers and (iv) datasets used. Additionally, we present two mappings: (i) mapping with mental disorders, DL techniques and additional used biomarkers and (ii) mapping with the nature of the disorder detection (diagnosis/prognosis), mental disorders and DL techniques. With those mapping, we will show what is already done and what could be done is this field. 
With the millions of people who are affected with a mental disorder in our mind, we have done this mapping to motivate to the research community to investigate in this field of study by clearly showing which are the most active topics as well as exposing some future research directions. In the same manner, we provide some guidelines for future studies.

\section{Methodology}

A Systematic Mapping Study (SMS) is a secondary study which reviews primary research with the aim of identifying research literature gaps in the field of interest (Petersen et al. 2008; Budgen et al. 2008; Kitchenham, Budgen, and Brereton 2011). Other secondary studies can be used to carry out reviews such as systematic literature review (Kitchenham and Charters 2007) but we have chosen SMS because of the clearer visual summary that this kind of study provides.

This work follows the SMS methodology proposed in (Petersen et al. 2008). However, we have applied a slightly different approach in order to provide more details and deeper understanding. Indeed, instead of reading only the papers' abstract and conclusion, a full read has been done because the shortage of works in our field of interest. The stages of the SMS are:

1. Definition of Research Questions. These are the questions that guide the work.

2. Conduct Search. Identification of the primary studies in databases through querying.

3. Screening of Papers. A inclusion and exclusion criteria are used to keep relevant papers according the work target.

4. Keywording of full text. A set of keywords and concepts is extracted of each paper while reading. These concepts could be put together into categories. Finally all keywords must be grouped. This enables us to relate all the works giving a global view and to put each one in context with regards to the rest. Keywording in the methodology proposed by (Petersen et al. 2008) is done by reading abstracts, but we have carried out with a full read because the low number of works present in out field of interest.

5. Data Extraction and Mapping of Studies. Frequency analysis of the keywords enables us to identify which categories has been exploited in the past on the topic and hence identify gaps and future research directions.

\subsection{Definition of Research Questions}

As we have to collect works which use EEG data with DL to detect or predict illness in patients, we propose the following four research questions:

- RQ1: Which mental disorders have already been diagnosed and prognosed by means of EEG and DL?

- RQ2: Which DL techniques have already been applied for diagnosing or prognosing mental disorders by using EEG data as input?

- RQ3: Which other biometric data is combined with EEG?

- RQ4: Which is the source of the datasets? 
Table 1: Queries made for each database

\begin{tabular}{|c|c|}
\hline Database & Query \\
\hline Scopus & $\begin{array}{l}\text { TITLE-ABS-KEY ( ( *eeg* OR electroencephalogra* ) AND } \\
\text { ( diagnos* OR prognos* ) AND "deep learning") }\end{array}$ \\
\hline Web of Science & $\begin{array}{l}\mathrm{TS}=\left(\left({ }^{*} \mathrm{eeg}^{*} \text { OR electroencephalograp* }\right) \text { AND ( diagnos* }\right. \\
\text { OR prognos* }) \text { AND "deep learning" })\end{array}$ \\
\hline IEEE Xplore & $\begin{array}{l}\text { "All Metadata":*eeg* OR electroencephalograp*) AND } \\
\text { ("All Metadata":diagnos* OR prognos*) AND ("All Meta- } \\
\text { data":"deep learning") }\end{array}$ \\
\hline ScienceDirect $^{\mathrm{a}}$ & $\begin{array}{l}\text { ( ( eeg OR electroencephalography ) AND } \\
\text { sis OR prognosis ) AND "deep learning" ) }\end{array}$ \\
\hline $\mathrm{ACM}$ & 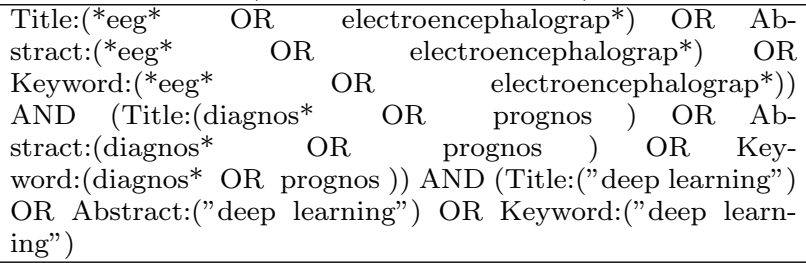 \\
\hline
\end{tabular}

${ }^{\text {a }}$ Wildcard characters are not supported in this database

\subsection{Conducted Search}

The following terms were used to assemble the set of works which will be used in order to answer our research questions:

- EEG

$-\mathrm{DL}$

- diagnosis

- prognosis

These terms were combined to assemble the query EEG and DL and (diagnosis or prognosis). The search was carried out in five well-known public databases, namely Scopus, Web of Science, IEEE Xplore, ScienceDirect and ACM Digital Library. In Table 1, it is shown the composed queries for each database according to each search engine syntax. These queries were carried out by using the following text fields: title, abstract and keywords. A total of 341 works were obtained. In the next subsection we defined an inclusion and exclusion criteria to obtain only relevant works.

\subsection{Screening of Papers}

Once the initial collection of works has been compiled, it is necessary to establish inclusion and exclusion criteria to keep only those works related to our research questions. Table 2 shows our defined criteria. Within the inclusion criteria, i1 is relevant because only few papers were found before 2016. That is because such year could be considered as the the upswing of DL (mainly, due to the introduction of GPU parallel processing) and we considered that the algorithms and techniques used prior to 2016 were not relevant for our goal. The criterion i2 was selected to keep only the most relevant works and $i_{4}$ because the followed methodology advised so (Petersen et al. 2008). Relative to the exclusion criteria, e1 ensure that 
Table 2: Inclusion and exclusion criteria used to keep relevant papers.

\begin{tabular}{|c|c|c|c|}
\hline \multicolumn{4}{|c|}{ criteria } \\
\hline \multicolumn{2}{|r|}{ Inclusion } & \multicolumn{2}{|r|}{ Exclusion } \\
\hline$i 1$ & Date of publish between 2016 to $2020-05-28$ & $e 1$ & No EEG signal used \\
\hline$i 2$ & Journal paper & $e^{2}$ & No mental disorder associated \\
\hline$i 3$ & English-written works & e3 & No deep learning \\
\hline$i_{4}$ & Primary study & & \\
\hline
\end{tabular}

we selected works where EEG is used in standalone form or combined with other biometric signals. Hence $e 1$ criteria is related to all of our four RQs showed in Section 4.1. The $e 2$ is used due to RQ1, we only want to keep works where mental disorders are treated. Finally $e 3$ is applied because of RQ2, and since we stick to field of artificial intelligence, that is, DL.

As shown in Figure 1, after removing duplicate works by using DOI, inclusion and exclusion criteria from Table 2 were applied. The criteria $i 4$, e1, e2 and e3 were carried out manually after reading the title and abstract of the 97 candidate works. At the end of this process a total of 46 studies were carefully selected to accomplish this work. For a comprehensive list with the selected papers and their extracted features, see Appendix B.

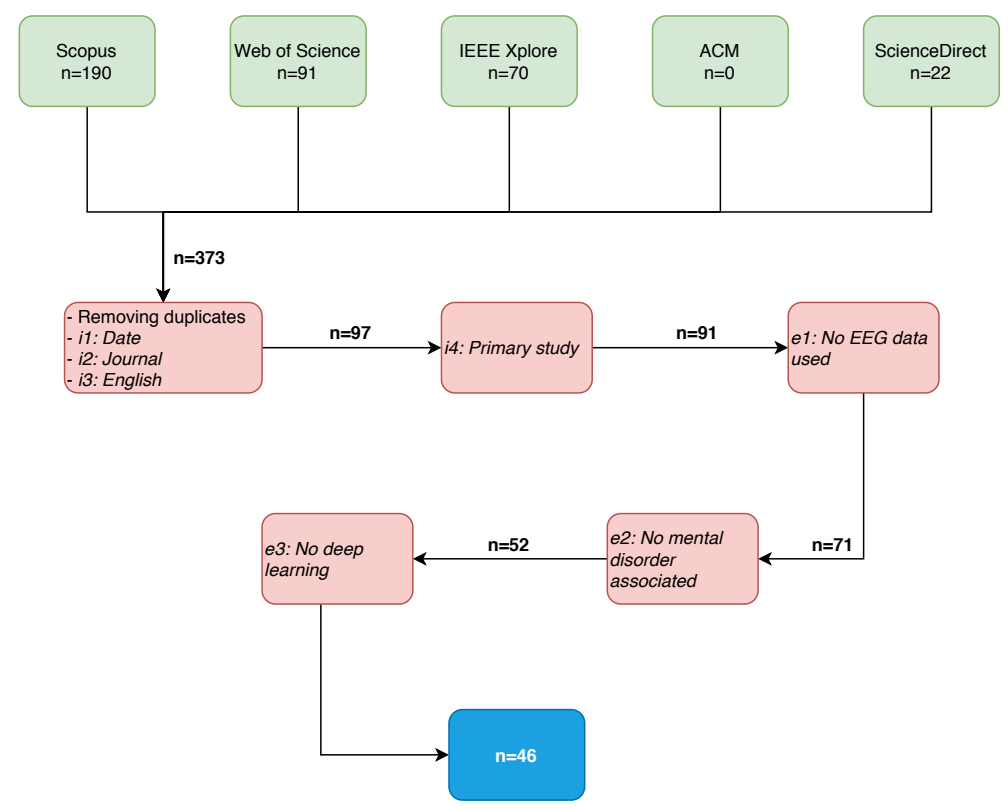

Fig. 1: Procedure to select works for mapping 
4.4 Keywording of full text

Following the methodology, prior to answering the RQs (see Section 4.1), it is fundamental to define for each RQ a set of keywords which are extracted by reading the papers. In our analysis, we firstly made a full lecture for each of the 46 selected papers where we extracted all the keywords that were considered as relevant to answer the RQs. Because of the number of keywords and the high granularity of them, we then defined groups of related keywords called categories. Finally each category is associated with the related RQ. The discovered keywords and categories are as follows:

RQ1.- After a full read of the selected works, we have collected a set of 23 keywords (see Table 3) which we will use to answer the RQ. Each of these keywords correspond to a mental disorder. A brief explanation of the gathered mental disorders is presented in the following: Attention Deficit Hyperactivity Disorder (ADHD) is a neuropsychiatric disorder characterized by inattentively, hyperactivity and impulsive behaviour which has high prevalence in children and adolescents (Kieling and Rohde 2012); Autism Spectrum Disorder is a developmental disorder that affects communication and social interaction by the presence of repetitive patterns and behaviours (Johnson et al. 2007); Coma is a prolonged state of unconsciousness where a person is alive but unable to move or interact with his or her environment (Jonas et al. 2019); Creutzfeldt-Jakob disease is a fatal and rapid degenerative brain disorder (Zerr et al. 2009) whose first symptoms are poor coordination, memory problems or impaired vision; Dementia is the progressive loss of cognitive functions that avoid to develop everyday activities; Alzheimer's Disease is the most common cause of dementia type (Ferri et al. 2005); Dementia with Lewy bod$i e s$ is a common form of dementia characterized leading in thinking problems and fluctuations in the level of consciousness (Weisman and McKeith 2007); Rapidly Progressive Dementia whose speed of patient's deterioration is over months, weeks or days (Geschwind et al. 2008); Depression is a serious mood disorder that affect negatively in sleeping, eating or working (Organization 2001); Fast Ripples are high frequency signals that can be recorded by EEG and has been studied as biomarker of Epilepsy (Bernardo et al. 2018); Epilepsy causes frequent seizures which is a abnormal electrical activity (Fisher et al. 2005); Parkinson's Disease is a progressive neurological disorder that cause not voluntary and uncontrollable movements in the body (Jankovic 2008); Schizophrenia is a mental disorder that interrupts the normal thinking, speech, feelings and behaviours (Andreasen 1982); Insomnia is a sleep disorder that can make it difficult to fall asleep (Roth 2007); Apnea is a sleep disorder where the patient has pauses in breathing (Young, Peppard, and Gottlieb 2002); Limb Movements makes the patient moves limbs involuntarily and during sleep (Picchietti and Winkelman 2005); Sleep Arousals are short interruptions in the sleep (Halász et al. 2004). From 23 keywords, we determined 10 mental disorder categories (see Table 3). Collapsing specific mental disorders into a category will enable us to obtain a high-level vision of the spectrum of disorders that have been diagnosed and prognosed with EEG and DL.

RQ2.- These 18 keywords relative to DL algorithms were extracted (see Table 4): Multilayer Perceptron $(M L P)$ is composed by one input layer, one or more hid- 
den layers and one output layer (Rumelhart, Hinton, and Williams 1986). If the network has more than one hidden layer then can be called Deep Neural Network (DNN); Convolutional Neural Network (CNN) uses the convolution operation in one or more layers where a convolution kernel is applied to the input data to produce a feature map. These networks can learn spatial features from the input data (LeCun et al. 1998); Fast Region-based Convolutional Network (Fast $R-C N N)$ develops object detection with high accuracy and less training time consumption because of the low number of parameters (Girshick 2015); Recurrent Neural Network (RNN) is a DNN which includes feedback connections capable to learn from sequence data (Rumelhart, Hinton, and Williams 1986); Long-Short Term Memory (LSTM) is a RNN that uses LSTM cells for solving the vanish gradient problem, performing better, taking less time to train and detecting long-term dependencies (Hochreiter and Schmidhuber 1997); Gated Recurrent Unit (GRU) is a simplification of the LSTM cell (Cho et al. 2014); Bidirectional LSTM not only preserve information of the input sequence from the past (backward) but also from the future (forward) (Graves and Schmidhuber 2005); RCNN and C-LSTM are hybrid architectures called Recurrent Convolutional Neural Network and Convolutional Long-Short Term Memory respectively, which are composed by a CNN model followed by a RNN model, these networks can learn spatio-temporal features from the input data (Zhou et al. 2015); Autoencoder is an neural network capable to create a deterministic low dimensional representation of the input vector (Bourlard and Kamp 1988); Stacked Autoencoder is an Autoencoder with more than one hidden layer (Hinton and Salakhutdinov 2006); Denoising Autoencoder $(D A E)$ differs that is trained with corrupted inputs, the result is a learned low dimensional representation more robust to noise (Vincent et al. 2010); Multimodal Denoising Autoencoder ( $M D A E$ ) is capable to learn a join representation from the input data that come from multiple modalities (Poirson and Idrees 2013); Convolutional Autoencoder ( $C A E$ ) can learn a low dimensional latent representation preserving spatial locality of the input data (Masci et al. 2011); Convolutional Variational Autoencoder (Convolutional VAE) is a probabilistic Autoencoder that can generate new instances similar to the training set (Kingma and Welling 2014) preserving spatial locality as well; Restricted Boltzmann Machines $(R B M)$ is a type of generative model that learns a probability distribution from its inputs and has not connections between visible or between hidden units (Smolensky 1986); Wasserstein Generative Adversarial Network ( $W G A N)$ is a generative model that involves two neural networks called generator, that produce fake noise samples from training data, and discriminator, that have to differentiate if the sample is real or a fake made by the generator, and uses Wasserstein distance for measuring model performance in order to avoid the vanishing gradient problem (Arjovsky, Chintala, and Bottou 2017); Siamese Neural Network SNN are two identical networks where comparing their encoding outputs for two given inputs, is returned the degree of similarity of these two inputs (Bromley et al. 1994). Once all keywords were put together, 13 categories were defined (see Table 4). That are: $A u$ toencoder, C-LSTM, CAE, CNN, Convolutional VAE, GRU, LSTM, MLP, RCNN, $R B M, R N N, S N N$ and $W G A N$. We only collapsed those keywords which, as far as we known, are DNN related ones. 
Table 3: Keywords and categories extracted for the RQ1

\begin{tabular}{|c|c|}
\hline Keyword & Category \\
\hline ADHD & ADHD \\
\hline Autism Spectrum Disorder & Autism \\
\hline Coma & Coma \\
\hline Creutzfeldt-Jakob disease & \multirow{5}{*}{ Dementia } \\
\hline Alzheimer's Disease & \\
\hline Dementia with Lewy bodies & \\
\hline Rapidly Progressive Dementia & \\
\hline Dementia & \\
\hline Unipolar Depression & \multirow{4}{*}{ Depression } \\
\hline Major Depressive Disorder & \\
\hline Mild Depression & \\
\hline Depression & \\
\hline Fast Ripples & \multirow{4}{*}{ Epilepsy } \\
\hline Idiopathic Generalized Epilepsy & \\
\hline Epileptic Seizure Detection & \\
\hline Epileptic Seizure Prediction & \\
\hline Parkinson's Disease & Parkinson's Disease \\
\hline Schizophrenia & Schizophrenia \\
\hline Insomnia & \multirow{5}{*}{ Sleep disorder } \\
\hline Sleep disorder & \\
\hline Apnea & \\
\hline Limb Movements & \\
\hline Sleep Arousals & \\
\hline
\end{tabular}

RQ3.- We have found eight keywords corresponding to different signals that were obtained in conjunction with EEG (see Table 5): Abdomen belt, Airflow and Chest belt capture respiratory signals; Electrocardiogram $(E C G)$ measure the electrical signals generated by the heart; Eye movement $(E M)$ data can be used to detect eye blinks and clean EEG signals; Electromyography $(E M G)$ measure the electrical activity produced by skeletal muscles; Electrooculography (EOG) capture the eye movements measuring the standing corneal-retinal potential; Oxygen Saturation (SaO2) measure by blood analysis.

RQ4.- Thirteen keywords were defined for RQ4 which compounds the same categories (see Table 6). Category Ad-hoc refers to a datasets made purposely for the work and Other refers to the use of third-party EEG data coming from other studies.

In the next section, we will answer the RQs by doing frequency analysis of the related categories and also, we will provide additional information extracted from the full read done of each work.

\subsection{Data Extraction and Mapping of Studies}

For answering the proposed RQs in Section 4.1, we carried out a frequency analysis of the categories defined above. It is worth noticing that a paper can involve more than one category for an attribute, e.g. there are papers where more than a DL algorithm, additional biometric data or dataset was used. In the same manner, 
Table 4: Keywords and categories extracted for the RQ2

\begin{tabular}{|c|c|}
\hline Keyword & Category \\
\hline Autoencoder & \multirow{4}{*}{ Autoencoder } \\
\hline Stacked Autoencoder & \\
\hline DAE & \\
\hline MDAE & \\
\hline C-LSTM & C-LSTM \\
\hline $\mathrm{CAE}$ & $\mathrm{CAE}$ \\
\hline $\mathrm{CNN}$ & \multirow{2}{*}{$\mathrm{CNN}$} \\
\hline Fast R-CNN & \\
\hline Convolutional VAE & Convolutional VAE \\
\hline GRU & GRU \\
\hline Bidirectional LSTM & \multirow{2}{*}{ LSTM } \\
\hline LSTM & \\
\hline MLP & MLP \\
\hline $\mathrm{RCNN}$ & $\mathrm{RCNN}$ \\
\hline RBM & RBM \\
\hline RNN & RNN \\
\hline SNN & SNN \\
\hline WGAN & WGAN \\
\hline
\end{tabular}

Table 5: Keywords and categories extracted for the RQ3

\begin{tabular}{|l|}
\hline Keyword Category \\
\hline Abdomen belt \\
\hline Airflow \\
\hline Chest belt \\
\hline ECG \\
\hline EM \\
\hline EMG \\
\hline EOG \\
\hline SaO2 \\
\hline
\end{tabular}

Table 6: Keywords and categories extracted for the RQ4.

\begin{tabular}{|l|}
\hline Keyword Category \\
\hline Ad-hoc \\
\hline Bonn University (Andrzejak et al. 2001) \\
\hline CHB-MIT (Shoeb 2009) \\
\hline Freiburg University (University of Freiburg 2003) \\
\hline $\begin{array}{l}\text { Lomonosov Moscow State University (Gorbachevskaya and } \\
\text { Borisov 2002) }\end{array}$ \\
\hline Kaggle $^{\mathrm{a}}$ \\
\hline Other \\
\hline PhysioNet/CinC 2018 Challenge \\
\hline Predict \\
\hline Sleep Heart Health Study (SHHS) (Dean et al. 2016) \\
\hline Temple University (Obeid and Picone 2016) \\
\hline UCI $^{\mathrm{d}}$ \\
\hline
\end{tabular}

${ }^{\text {a }}$ https://www.kaggle.com/

b https://physionet.org/content/challenge-2018/1.0.0/

${ }^{c}$ http://predict.cs.unm.edu/downloads.php

d https://archive.ics.uci.edu/ml/index.php 
some studies do not provide information for some analyzed attributes such as sampling frequency or the number of EEG channels, among others. Thus, in the following analysis, the reader will notice how the sum of papers may be higher or lower (because the study do not provide information), that the number of selected papers $(n=46)$. In Appendix B is provided the extracted information from the selected studies allowing the reproducibility of our results. In overall, the number of the published works growth each year (see Figure 12) being China, USA and Malaysia the countries where more studies are produced (see Figure 13). The frequency analysis for the RQs are as follows:

RQ1.- Nine mental disorders have been diagnosed or prognosed with DL (see Figure 2a). The predominant disorder found was epilepsy, representing the $47.83 \%$ (22 papers) of the total of studies, followed by depression with a $15.22 \%$ (7 papers) and schizophrenia with $8.7 \%$ (4 papers). The next disorders correspond to sleep disorder, dementia (3 papers) and ADHD with a $6.52 \%$ (3 papers) for each one, Coma with $4.35 \%$ (2 papers) and finally Parkinson's disease and autism appearing in (Ruffini et al. 2019; Ali et al. 2020) respectively. Concerning to the performance achieved by classifying each mental disorder, we have extracted the accuracy for those studies which made a more-reliable intra-subject validation, that is, the test set only contains patients not used in the training set. In the same manner, there is not possible to carry out an objective performance comparison between studies because (i) there is not a reference dataset for each mental disorder and (ii) there is not a common set of metrics used in the studies to measure the performance (see Section 5 for more details). Hence, the following information is provided as a guideline only. The median accuracy by mental disorders is as follows: sleep disorder $90.1 \%$, schizophrenia $88.13 \%$, epilepsy $87.84 \%$, comma $87.04 \%$, depression $82.74 \%$, Parkinson's disease $81 \%$ and ADHD $83 \%$ (see Figure 3a). For dementia and autism, the studies carry out inter-subject validation. On the other hand, we have classified the studies in two classes (see Figure $2 \mathrm{~b}$ ): diagnosis and prognosis. Most of the studies perform diagnosis of the mental disorders with a $69.64 \%$ (39 papers) with $23.21 \%$ for prognosis (13 papers). It is worth noting that there are 6 studies (Thara, PremaSudha, and Xiong 2019; Liang et al. 2019; Wei et al. 2018; Acharya et al. 2018b; Hussein et al. 2019; Khan et al. 2018) where we considered that both, diagnosis and prognosis, was carried out.

RQ2.- CNN is the most widely used DL algorithm (see Figure $3 \mathrm{~b}$ ) with a $48.58 \%$ (28 papers), following by C-LSTM with a $10.34 \%$ (6 papers), LSTM with a $8.62 \%$ (5 papers) and AE with a $5.17 \%$ (3 papers). After, we can find RNN, MLP, GRU, CAE and Bidirectional LSTM with a $3.45 \%$ (2 papers) for each one. The DL algorithms with less frequency are WGAN, SNN, RBM, RCNN, DAE and Convolutional VAE used in (Wei et al. 2019; Calhas, Romero, and Henriques 2020; Bi and Wang 2019; Biswal et al. 2018; Yuan et al. 2019; Abdelhameed and Bayoumi 2019) respectively. It is clear that Convolutional and Recurrent networks are the most used techniques, with the hybridization between these two models, that is, C-LSTM standing in third place. Because of epilepsy is the most frequent mental disorder, we have repeated this analysis after removing studies which involve this mental disorder. We noticed how the distribution of DL algorithms remain similar. Consequently, our analysis is not biased. For those studies that provide details, some characteristics of their DL algorithms were extracted: 
- The mean of the number of layers is 8.5 with a standard deviation of 4.81 (see Figure 4a).

- The activation functions used are: softmax appears in the $45.28 \%$ (24 papers), second is Rectified Linear Unit (ReLU) with a 30.19\% (16 papers) and third is sigmoid with a $9.43 \%$ ( 5 papers). Next are hyperbolic tangent (Tanh) and Leaky ReLU present in a 5.66\% (3 papers) for each one. Finally Exponential Linear Unit (ELU) and linear activation functions were only used in (Vahid et al. 2019) (see Figure 4b).

- Dropout to avoid overfitting is used in 52.17\% (22 papers) and Batch Normalization, which avoids the vanish and explode gradient problems, is less used, thus appearing in the $19.57 \%$ (9 papers) (see Figure 4c).

- In term of optimizers, Adam is the most widely used with a $78.79 \%$ (26 papers) following by Stochastic Gradient Descent (SGD), RMSprop and Adadelta with a $6.06 \%$ (2 papers) for each one. Finally Nadam only appears in (Chen, Song, and Li 2019) (see Figure 4d).

As for the type to the metrics used to measure the performance (see Figure 5a), the four most used are accuracy with a $29.13 \%$ (37 papers), sensitivity with a $23.62 \%$ (30 papers), specificity with a $17.32 \%$ (22 papers) and ROC-AUC with a $11.02 \%$ (14 papers). The rest of the metrics are as follows: precision with a $7.87 \%$ (10 papers), F1-score with a $5.51 \%$ ( 7 papers), false positive rate with a $3.15 \%$ (4 papers), precision-recall curve (PRC) with a $1.57 \%$ (2 papers) and false negative rate with $0.79 \%$ ( 1 paper). On the other hand, $39.13 \%$, that is, 18 papers have measured the performance of the proposed DL algorithm with a intra-subject validation (see Figure 5b).

RQ3.- As we explained above, we have found eight additional signals processed together with EEG signals (see Table 6) in only four different papers (8.7\%). EMG is the most frequent one which has been used in (Wei et al. 2019; Biswal et al. 2018; Warrick, Lostanlen, and Nabhan Homsi 2019). Abdomen belt, Airflow, Chest Belt and $\mathrm{SaO} 2$ were used in (Biswal et al. 2018; Warrick, Lostanlen, and Nabhan Homsi 2019). ECG and EOG appear in (Wei et al. 2019; Warrick, Lostanlen, and Nabhan Homsi 2019). Finally, EM was used in (Zhu et al. 2019).

RQ4.- There are 10 public EEG datasets present in the $50.98 \%$ of the studies. Ad-hoc datasets which were built for the paper purpose or reused from the author's previous study are employed in the $43.14 \%$ (see Figure $7 \mathrm{a}$ ). A third case named Other refers to the use of third-party EEG data coming from other studies which constitutes the 5.88\%. In five works (Abdelhameed and Bayoumi 2019; Wen and Zhang 2018; Khan et al. 2018; Biswal et al. 2018; Fürbass et al. 2020) two datasets were used. In the Figure $7 \mathrm{~b}$, it is showed the frequency in the use of datasets by their source (ad-hoc, public or other) and mental disorder. Only in epilepsy, schizophrenia, sleep disorder and depression there are studies where a public dataset has been used. Moreover, in the first three mental disorders, the number of studies carried out with public datasets is greater or equal to those performed with ad-hoc datasets. On the other hand, regarding to the number of channels used to record EEG signals, 19 is the most common number with a $21.05 \%$ (8 papers) and $256 \mathrm{~Hz}$ is the most used sample rate with a $35 \%$ (14 papers). See Figures 8 and 9 . 


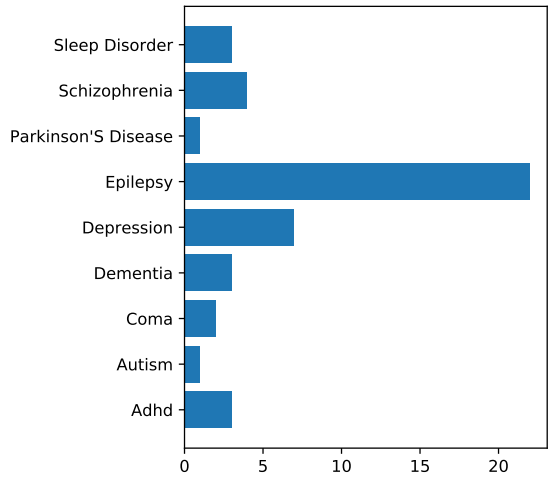

(a)

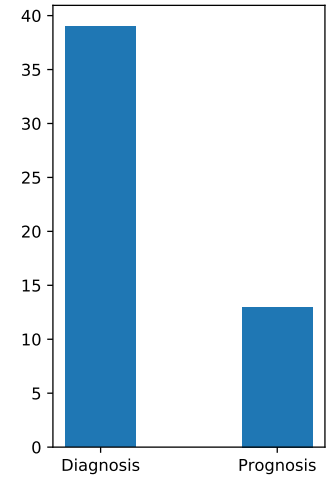

(b)

Fig. 2: (a) Frequency of defined mental disorders categories in selected works and (b) frequency of nature of the disorder detection in selected works

Finally, as proposed in the methodology (Petersen et al. 2008), we have carried out two visualizations to map the RQ1, RQ2 and RQ3. We consider that doing these mapping where RQs are combined, enables us to detect gaps where new research works could be carried out. In the following, the relationship among RQ1 with RQ2 and RQ3 will be presented:

- Mapping of mental disorders with DL (mapping of the RQ1 and RQ2). Epilepsy is the mental disorder where most of the DL techniques has been used: CNN, RNN, C-LSTM, Bidirectional LSTM, CAE, Convolutional VAE, DAE, GRU, LSTM and WGAN. In second place is dementia with four algorithms: CNN, AE, MLP, RBM. With three DL techniques are sleep disorder (MLP, Bidirectional LSTM and RCNN), Parkinson's disease (CNN, GRU, LSTM), schizophrenia (CNN, GRU, LSTM) and depression (CNN, AE, C-LSTM). In ADHD only CNN and RNN have been studied. Finally in coma and autism CNN is the only technique employed. See Figure 10.

- Mapping of Mental disorders and other biometric data (mapping of the RQ1 and $R Q 3$ ). In sleep disorder EEG data has been combined with ECG, EMG, EOG, Abdomen belt, Airflow, Chest belt and $\mathrm{SaO} 2$ signals, that is, 7 out 8 additional biometric data categories identified. In epilepsy, ECG, EMG and EOG has been used. At last with depression only appears with EM. See Figure 10.

- Mapping of mental disorder with the nature of the disorder detection (mapping of the RQ1). Diagnosis has been carried out for ADHD, autism, depression, schizophrenia, and sleep disorder. Prognosis has been done for coma and Parkinson's disease. With dementia and epilepsy, both diagnosis and prognosis, has been carried out. See Figure 11.

- Mapping of DL with the nature of the disorder detection (mapping of the RQ1 and RQ2). For diagnosis, only GRU cells has not been used. In prognosis there has been used AE, Bidirectional LSTM, C-LSTM, CNN, LSTM, RNN and GRU. See Figure 11. 


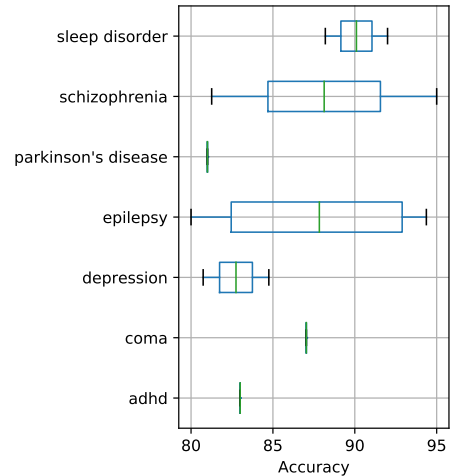

(a)

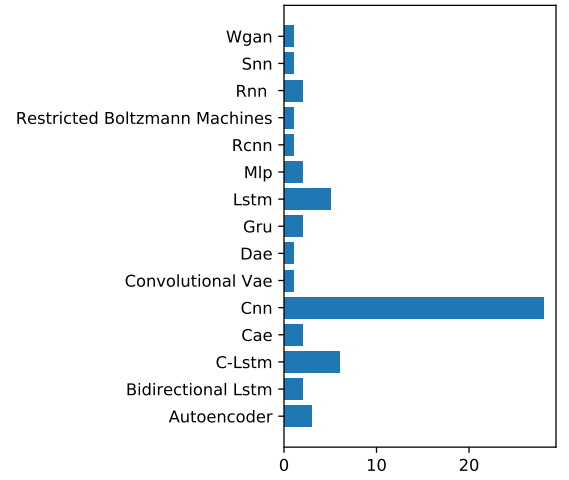

(b)

Fig. 3: (a) Accuracy achieved by mental disorder (only studies with intra-subject validation) and (b) frequency of DL techniques present in the studies

\section{Discussion}

In this section, we will discuss interesting insights discovered in this review and expose some issues we found out for those we will provide some insights in order to address them.

Related to mental disorders and DL techniques (see Figure 10), we can say that, without taking account epilepsy which is where most studies have focused, the rest of mental disorders present several gaps regarding to DL techniques application. Thus, these gaps indicate unexplored fields where new researches could be carried out, that is, processing EEG data with DL to diagnose or prognose mental disorders. In this sense, CNN is the most used DL architecture, while the rest of the algorithms are much less used. To the best of our knowledge, CNNs can extract spatial features from the input data but not features that take into account the sequentiality present in those data, i.e. EEG data. We consider that more efforts should be done with regards to the use of recurrent DL architectures to take advantage of the temporal component, i.e. using LSTM or GRU cells. Furthermore in (Liu et al. 2020) the hybrid architecture C-LSTM was compared with CNN and LSTM for seizure detection, obtaining C-LSTM the best performance. In our opinion this result should motivate to use C-LSTM in the new works. With this in mind, and since the no free lunch theorem establishes that there is no best algorithm to every problem, it should motivate us to use more than one algorithm when a new research work is done because we need (i) to achieve the best possible performance and (ii) to compare the performance of these algorithms in the diagnosis or prognosis of a specific illnesses.

When using CNNs, an important choice is how to sort EEG channels because adjacent channels will be processed together due to $2 \mathrm{D}$ convolution operation. Usually, EEG data is presented to CNNs as a 2D array, where the $\mathrm{X}$-axis is the time and the Y-axis represents the EEG channels (see Figure 14). To carry out 


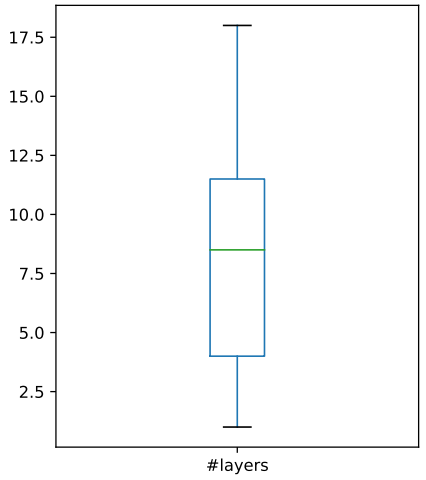

(a)

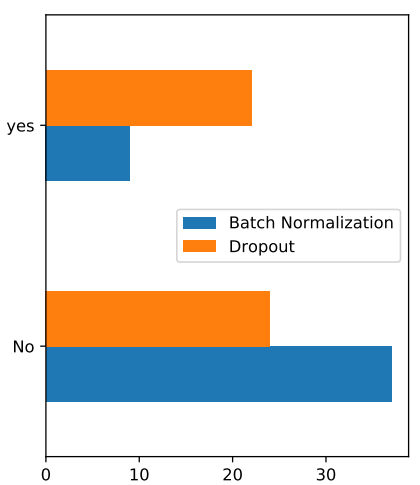

(c)

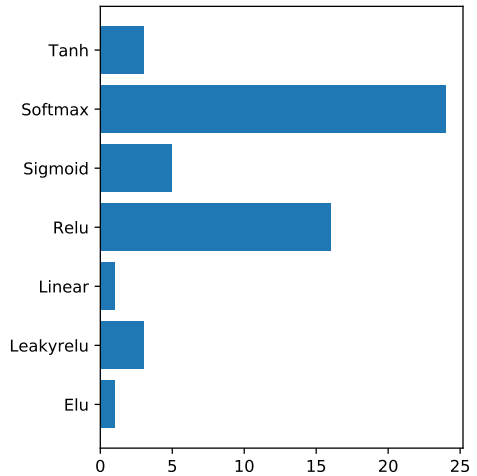

(b)

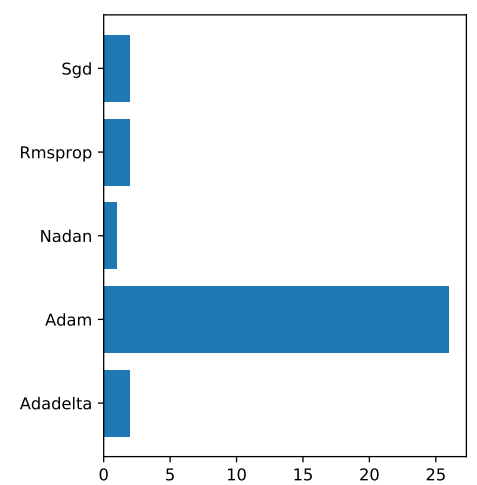

(d)

Fig. 4: (a) number of layers used for the DL algorithms, (b) frequency of the activation functions, (c) frequency of use of Batch Normalization and Dropout and (d) frequency of the optimizers.

a $2 \mathrm{D}$ convolution, a kernel with size $n x m$ needs to be defined, where $n$ is the number of consecutive "pieces" of time taken into account, and $m$ is the number of channels which are convoluted together. Hence, the order of the channels will condition the accuracy of the network. A common solution is to sort the channels regarding their correlations. In our opinion, it may not be a good solution because channels which are not correlated with another would be treated in an isolated manner and would not be put together with another channel with no correlation. To avoid this issue, we propose the following method: firstly split the EEG record (2D array) in several equal-sized 2D arrays (called records). Secondly, build a 3D array by appending all those arrays where the dimensions are X-time, Y-channels, Z-record. Finally, compound a 3D kernel to carry out 3D convolutions to process every channel in an isolated manner. In Figure 15 we show this process.

A major advantage of the use of DL is the possibility of using pre-trained networks to carry out a totally new but related task. This can save computing time 


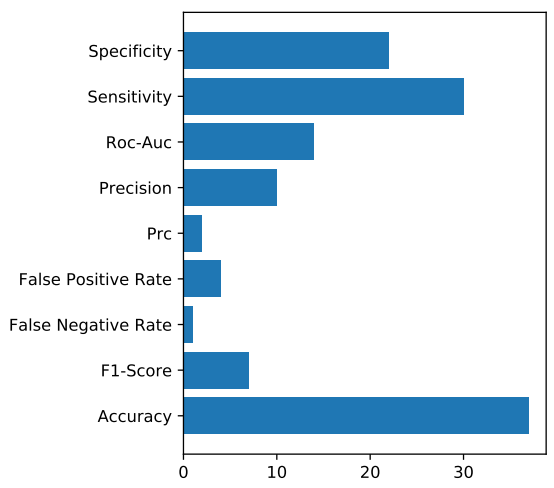

(a)

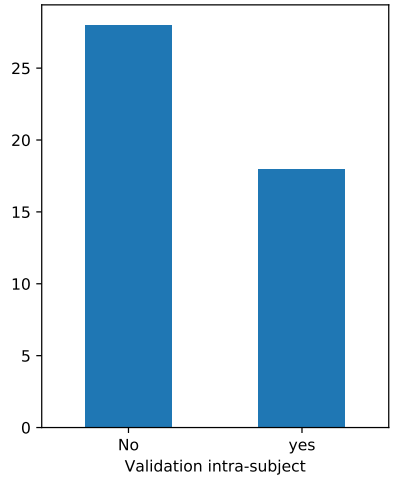

(b)

Fig. 5: (a) Frequency of the metrics used in the studies and (b) amount of studies where intra-subject validation is carried out.

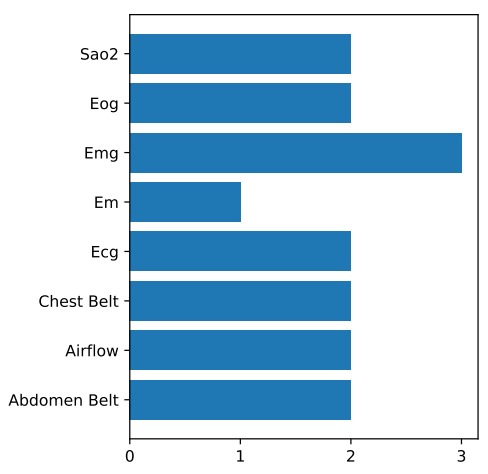

Fig. 6: Frequency of additional biosignals extracted while EEG recording

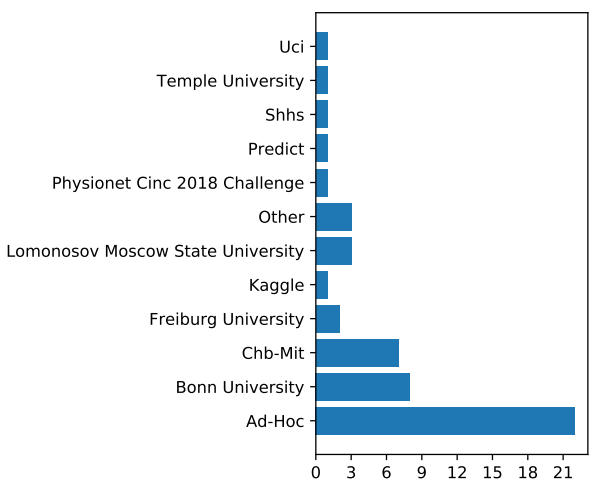

(a)

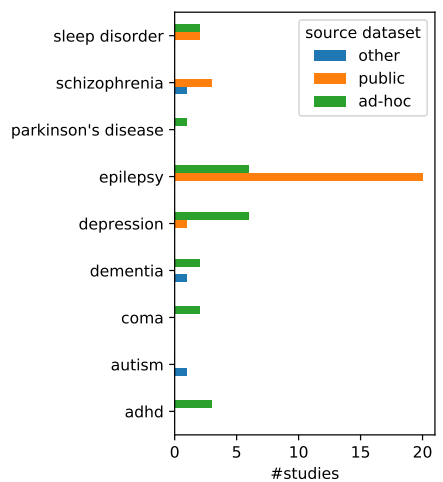

(b)

Fig. 7: (a) Frequency of the used EEG datasets and (b) frequency in the use of datasets by their source and mental disorder. 


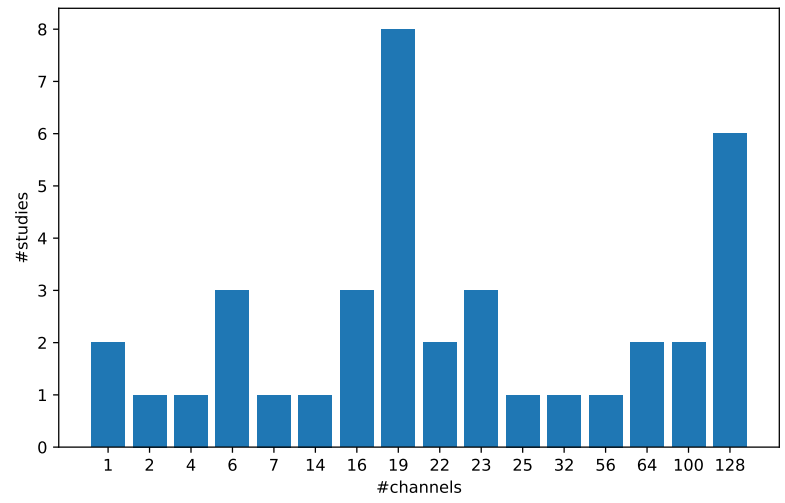

Fig. 8: Frequency of number of channels used to record EEG signals

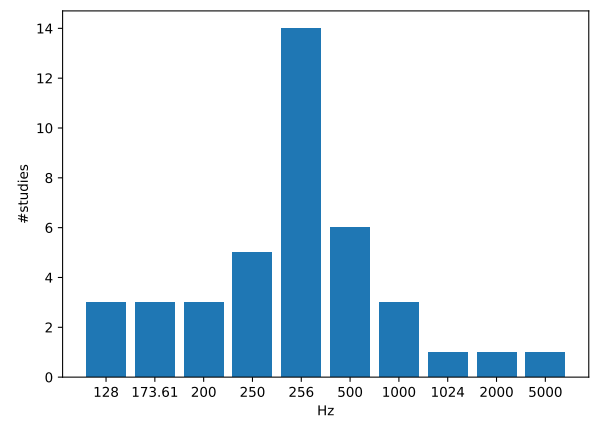

Fig. 9: Frequency of the sampling rate used to record EEG signals

and enables us to perform the training stage with less data. This concept is called transfer learning (Yosinski et al. 2014). In our opinion, using transfer learning to process EEG data should be used when the number of EEG records for training is low, e.g. due to the lack of patients to accomplish EEG records. An example of application could be training a network to classify patients with sleep disorders using available public datasets (see Table 6), and then training a classifier to diagnosis another mental disorder, e.g. depression, by using transfer learning over this pre-trained network.

Considering the mental disorders which have been diagnosed or prognosed with DL (see Figure 2a), and according to (Wittchen et al. 2011) where the most prevalent 12 month disorders in Europe 2010 were presented, we have identified a set of mental disorders with high prevalence which are not been diagnosed or prognosed by means of EEG and DL. Those mental disorders are: i) anxiety disorders which is the most prevalent 12-month disorder in Europe affecting to $61.5 \mathrm{M}$ of persons, ii) alcohol dependence $14.6 \mathrm{M}$, iii) post traumatic stress disorder $7.7 \mathrm{M}$, iv) personality disorders $6.3 \mathrm{M}, \mathrm{v}$ ) mental retardation $4.2 \mathrm{M}$, vii) obsessive compulsive disorder $2.9 \mathrm{M}$, viii) conduct disorder $2.1 \mathrm{M}$, ix) eating disorders $1.5 \mathrm{M}$ and $\mathrm{x}$ ) cannabis dependence $1.4 \mathrm{M}$. Because the amount of people affected and the promis- 


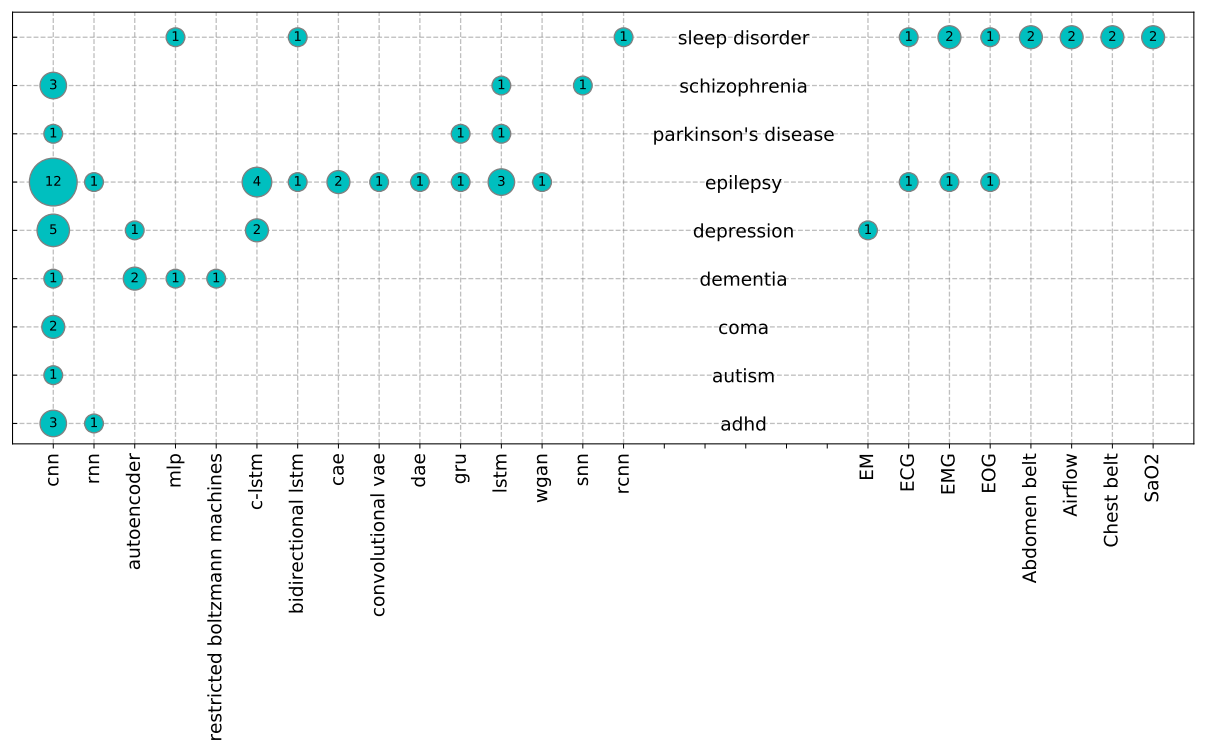

Fig. 10: Mapping with the frequency of studies combining categories from RQ1 with RQ2 and RQ3

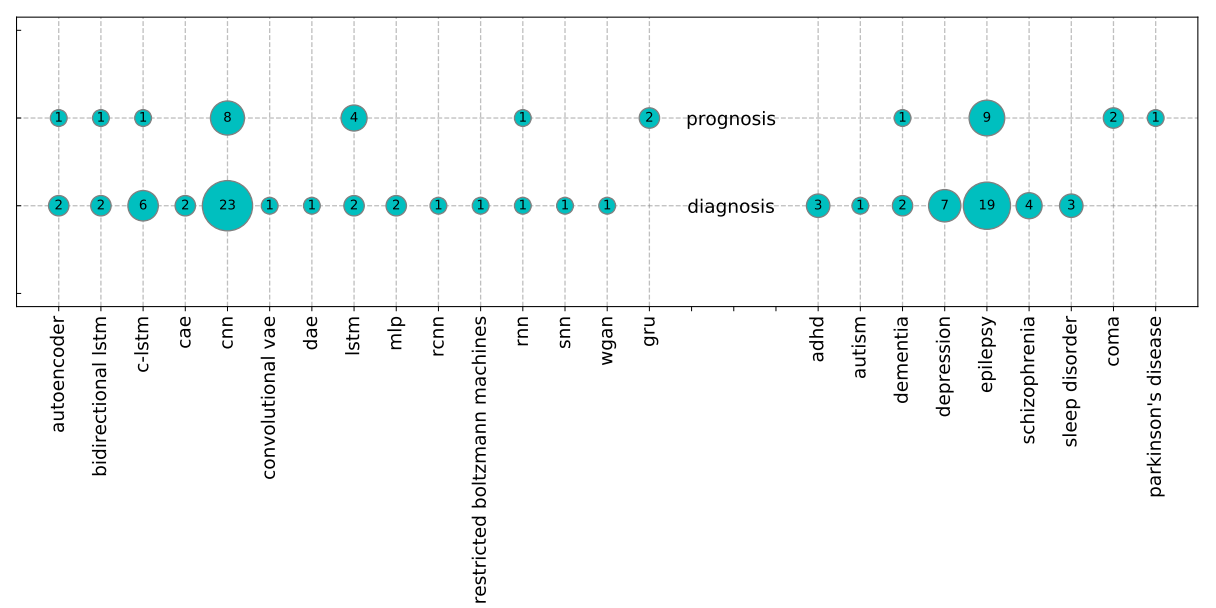

Fig. 11: Mapping with the frequency of studies combining the nature of the disorder detection (diagnosis, prognosis) with RQ1 and RQ2

ing results employing DL with EGG, efforts in diagnosis and prognosis of these mental disorders by means EEG and DL should be done. For a complete reference list of mental disorders see (Association et al. 2013).

Regarding to recording and processing other biometrical data alongside EEG, we can say that this is an open field, as we showed in our mapping (see Figure 10), 


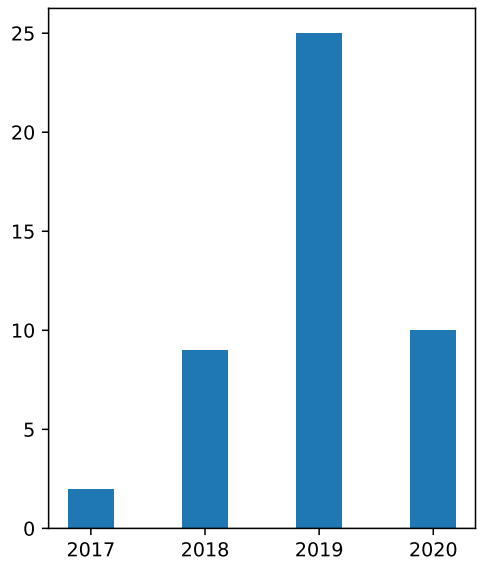

Fig. 12: Number of studies published per year until 2020-05-28

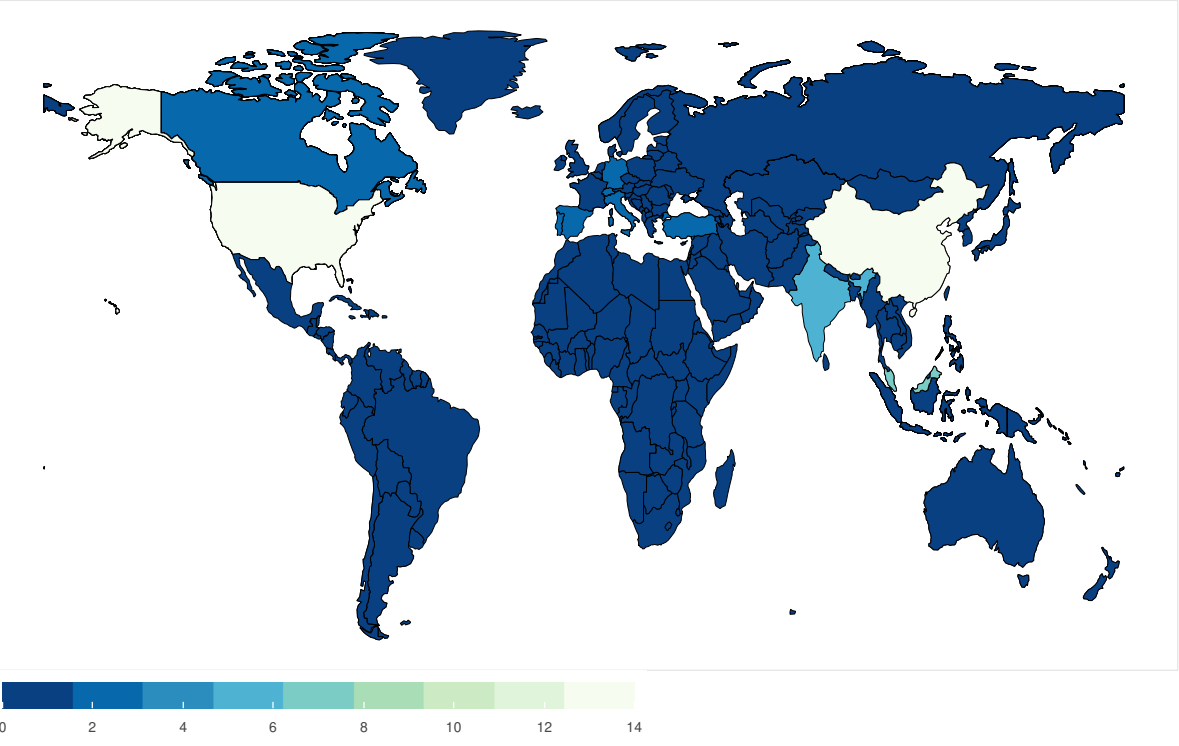

Fig. 13: Frequency of the countries for all appearing authors in the selected papers

only the $8.7 \%$ of the studies have used at least one additional kind of biometric data. In the same manner, only in the $33 \%$ of the found mental disorders (Sleep Disorder, Epilepsy and Depression), EEG has been combined with at least another additional biomarker. In this sense, (Zhu et al. 2019) showed promising results by joining EEG and EM to increase the performance of the classifier for mild depression recognition. In addition to the biometric signals used in the selected studies, there are previous works where other different signals were combined with EEG: in (Dupuy et al. 2014) EEG and electrodermal activity (EDA) was employed to 


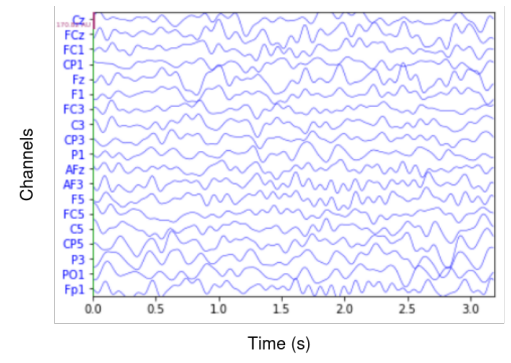

Fig. 14: Example of EEG record with 19 channels

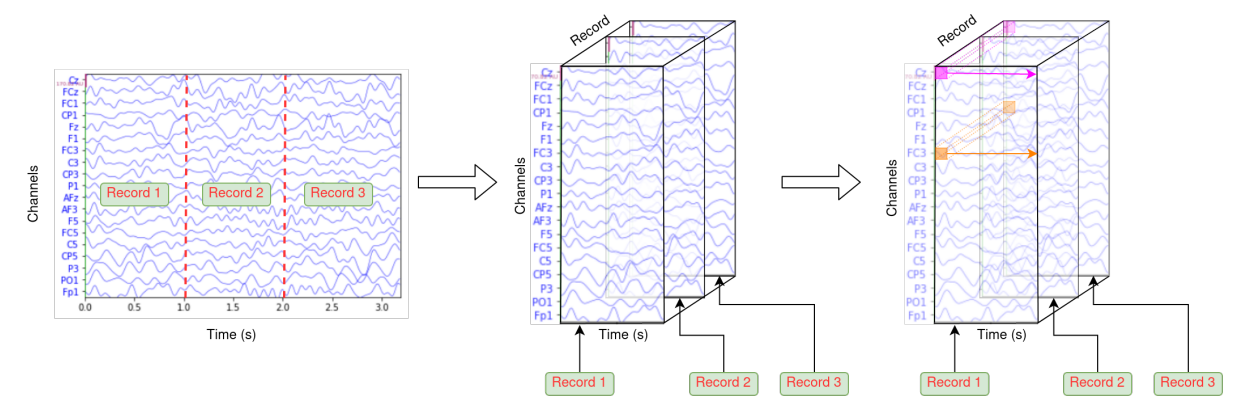

Fig. 15: Preprocessing EEG data to carry out 3D convolutions over every channel in an isolated manner. Pink and orange cuboids are two examples of 3D kernels convolutions.

explain ADHD symptoms. In (Gallagher et al. 2008), they used EEG with nearinfrared spectroscopy at the same time to locate the ictal onset zone in a epileptic patient. It is important to notice that, as (Zhu et al. 2019) mention, if some of these biometric signals are recorded, an accurate synchronization between these signals and EEG data is essential for simultaneous analysis.

DL are blackbox algorithms, that is, their predictions are not self-explanatory. Giving an explanation to the outcome can increase the confidence in their predictions by showing that EEG electrodes and their amplitude are relevant to classify a mental disorder. This is known as the inverse problem. Providing that explanations could help doctors to trust in DL-based tools. In (Shahin et al. 2017), it was used the Gradient-Weighted Class Activation Mapping (Grad-CAM) to identify which EEG features have more importance for the network to predict a favourable outcome. To explain the CNNs predictions SHAP (Lundberg and Lee 2017) can be used, which is a game-theory-based approach aimed at explaining ML algorithms. In their library (Lundberg and Lee 2016) two methods for explaining CNNs are available. For a complete review of explainable methods for DL refer to (Xie et al. 2020).

The validation stage enables us to know the performance of the proposed DL framework. As we showed, the only $39.13 \%$ of the works realized an intra-subject 
validation (see Figure 5b). This type of validation offers a realistic performance of the algorithm because the test set is composed by different never-seen subjects, which were not used for training. In contrast, an inter-subject validation offers an artificial high level of performance because it do not show how the algorithm will generalize its outcomes for a new patient. Hence, we encourage the use of intrasubject validation. Furthermore, it is important to emphasise the significance of being able to objectively compare the classification results among studies. This comparison enables us to know which are the algorithms that achieve the best performance by mental disorder. Unfortunately, only a preliminary comparison of results has been showed because of (i) there is not a common set of metrics used in the studies to measure the performance and (ii) there is not a reference dataset by mental disorder. In this respect, only in 4 out 9 mental disorders (epilepsy, schizophrenia, sleep disorder and depression) there is available a public dataset (see Figure $7 \mathrm{~b}$ ). It is worth noting that, these 4 mental disorders are the most common across the studies (see Figure 2a). Nevertheless, for future works (i) only intra-subject validation should be performed, (ii) a reference dataset should be created by mental disorder and (iii) a standard set of metrics should be used for objectively measure the performance.

With regards to the reproducibility of the results, we have found out that the majority of the studies do not share their source code, thus making their outcome non-reproducible. Besides, some of the works neither offer a good detailed explanation of the DL architecture nor presents the set-up process for the EEG data acquisition process, such as the layout used to place the electrodes, sample frequency, or subject characteristics. In the same manner, the captured EEG data rarely is shared but as we noticed, sometimes privacy restrictions do not allow researchers to make it publicly available. We consider that a detailed explanation of the selected patients, capturing EEG data process, preprocessing (if required), DL architecture and the type of training and validation used should be provided. Additionally, source code and data ideally should be shared. All of these pieces of information would allow the progress beyond the state of the art in this field progress.

Finally, we have to mention that a limitation of our study is that it is focused on diagnosis or prognosis of mental disorders, hence the results presented in our work could not be generalized to other fields where EEG is used.

\section{Conclusions}

There is a growing interest in using EEG to train DL algorithms for diagnosis and prognosis of mental disorders because of the promising results observed and the continuous improvements achieved by using DL techniques. In this work, a Systematic Mapping Study is presented for clearly knowing what is already done and what could be done within this field. Four RQs were proposed in Section 4.1 and four public databases were queried to retrieve relevant works. 46 primary studies were finally selected and after a full read, there were extracted relevant keywords which were grouped in categories to answer the RQs. 
The main results are as follows: (i) nine mental disorders categories which have been diagnosed or prognosed by using EEG data and DL, being Epilepsy the most common one, present in $47.83 \%$ of the works, (ii) 13 categories of DL algorithms, being CNN the most frequent appearing in $48.28 \%$ of the papers, (iii) eight additional biomarkers were combined with EEG data in $8.7 \%$ of the studies and (iv) in $50.98 \%$ of the works, it were used a public EEG dataset.

Additional discoveries were done by combining RQs: (i) epilepsy is the mental disorder where more DL techniques have been tried out and (ii) additional biomarkers have only been employed in sleep disorder, epilepsy and depression. Finally in the discussion section, insights and recommendations were done: (i) without taking account epilepsy, for the rest of mental disorders there are many gaps in the use of DL techniques. For future works, we recommend to focus in the use of LSTM and GRU cells, as well as the hybrid C-LSTM networks because of the continuous nature of the EEG signal, (ii) some of the most prevalent mental disorders such as anxiety, alcohol dependence, post traumatic stress, personality disorders, mental retardation, obsessive compulsive disorder, conduct disorder, eating disorders and cannabis dependence have not been diagnosed or prognosed by using DL and EEG, (iii) combining additional biomarkers with EEG is a promising approach to improve the classification results, yet more efforts should be done in this sense. Other recommendations were also provided: (i) explaining the DL model results can be achieved for some DL techniques by using of available frameworks. It can help in the adoption of DL techniques by providing the most relevant EEG channels for the model predictions, (ii) we encourage to perform intra-subject validation for getting a more realistic performance of the algorithms, thus avoiding artificial high accuracy, (iii) to objectively compare performance between studies, it is important to define a reference dataset by mental disorder, as well as the use of a standard set of metrics for measuring the DL performance, and (iv) we suggest paying more attention to ease the reproducibility of their results by providing more details of the selected patients, capturing EEG data process, preprocessing (if needed), DL architecture and the type of training and validation used as well as sharing both the source code and the recorded data in an anonymous manner.

As a limitation, our study is focused on diagnosis or prognosis of mental disorders. Hence the results presented in our work could not be generalized to other fields where EEG is used.

Acknowledgements This work has been funded by the ECLIPSE project (RTI2018-094283B-C32) from the Spanish Ministry of Science, Innovation and Universities.

\section{Conflict of interest}

The authors declare that they have no conflict of interest. 


\section{References}

Abdelhameed, Ahmed M. and Magdy Bayoumi (2019). "Semi-supervised EEG signals classification system for epileptic seizure detection". In: IEEE Signal Processing Letters 26.12, pp. 1922-1926. ISSN: 15582361. DOI: 10.1109/LSP. 2019. 2953870.

Acharya, U. Rajendra et al. (2012). "Automated diagnosis of normal and alcoholic EEG signals". In: International Journal of Neural Systems 22.3. ISSN: 01290657. DOI: 10.1142/S0129065712500116. URL: https ://www.researchgate. net/publication/236579449.

Acharya, U. Rajendra et al. (2018a). "Automated EEG-based screening of depression using deep convolutional neural network". In: Computer Methods and Programs in Biomedicine 161, pp. 103-113. ISSN: 18727565. DOI: 10.1016/ j . cmpb.2018.04.012. URL: https://doi.org/10.1016/j.cmpb.2018.04.012.

Acharya, U Rajendra et al. (2018b). "Deep convolutional neural network for the automated detection and diagnosis of seizure using EEG signals". In: Computers in Biology and Medicine 100, pp. 270-278. ISSN: 0010-4825. DOI: https: // doi . org / 10 . 1016/j . compbiomed . 2017 . 09 . 017. URL: http : / / www . sciencedirect.com/science/article/pii/S0010482517303153.

Ali, Nur Alisa et al. (2020). "Autism spectrum disorder classification on electroencephalogram signal using deep learning algorithm". In: IAES International Journal of Artificial Intelligence 9.1, pp. 91-99. ISSN: 22528938. DOI: 10.11591/ijai.v9.i1.pp91-99.

Andreasen, Nancy C. (1982). "Negative Symptoms in Schizophrenia: Definition and Reliability". In: Archives of General Psychiatry 39.7, pp. 784-788. ISSN: 15383636. DOI: 10.1001/archpsyc.1982.04290070020005.

Andrzejak, Ralph G. et al. (2001). "Indications of nonlinear deterministic and finite-dimensional structures in time series of brain electrical activity: Dependence on recording region and brain state". In: Physical Review E - Statistical Physics, Plasmas, Fluids, and Related Interdisciplinary Topics 64.6, p. 8. ISSN: 1063651X. DOI: 10.1103/PhysRevE.64.061907.

Arjovsky, Martin, Soumith Chintala, and Léon Bottou (2017). "Wasserstein GAN". In: arXiv: 1701.07875. URL: http://arxiv.org/abs/1701.07875.

Association, American Psychiatric et al. (2013). Diagnostic and statistical manual of mental disorders (DSM-5®). American Psychiatric Pub.

Ay, Betul et al. (2019). "Automated Depression Detection Using Deep Representation and Sequence Learning with EEG Signals". In: Journal of Medical Systems 43.7. ISSN: 1573689X. DOI: 10.1007/s10916-019-1345-y.

Baig, Muhammad Zeeshan, Nauman Aslam, and Hubert PH Shum (2020). "Filtering techniques for channel selection in motor imagery EEG applications: a survey". In: Artificial intelligence review 53.2, pp. 1207-1232.

Baloglu, Ulas Baran and Özal Yildirim (2019). "Convolutional long-short term memory networks model for long duration EEG signal classification". In: Journal of Mechanics in Medicine and Biology 19.1, pp. 1-21. ISSN: 02195194. DOI: 10.1142/S0219519419400050.

Bernardo, Danilo et al. (2018). "Visual and semi-automatic non-invasive detection of interictal fast ripples: A potential biomarker of epilepsy in children with tuberous sclerosis complex". In: Clinical Neurophysiology 129.7, pp. 1458-1466. 
ISSN: 18728952. DOI: 10.1016/j.clinph.2018.03.010. URL: https://doi . org/10.1016/j.clinph.2018.03.010.

Bi, Xiaojun and Haibo Wang (2019). "Early Alzheimer's disease diagnosis based on EEG spectral images using deep learning". In: Neural Networks 114, pp. 119 135. ISSN: 18792782. DOI: $10.1016 / \mathrm{j}$. neunet . 2019 .02 .005. URL: https : //doi.org/10.1016/j.neunet.2019.02.005.

Biswal, Siddharth et al. (2018). "Expert-level sleep scoring with deep neural networks". In: Journal of the American Medical Informatics Association 25.12, pp. 1643-1650. ISSN: 1527974X. DOI: 10.1093/jamia/ocy131.

Bourlard, Hervé and Yves Kamp (1988). "Auto-association by multilayer perceptrons and singular value decomposition". In: Biological cybernetics 59.4-5, pp. 291-294.

Bromley, Jane et al. (1994). "Signature verification using a" siamese" time delay neural network". In: Advances in neural information processing systems, pp. 737-744.

Budgen, David et al. (2008). "Using Mapping Studies in Software Engineering." In: PPIG. Vol. 8, pp. 195-204.

Calhas, David, Enrique Romero, and Rui Henriques (2020). "On the use of pairwise distance learning for brain signal classification with limited observations". In: Artificial Intelligence in Medicine 105.March, p. 101852. ISSN: 18732860. DOI: 10.1016/j .artmed.2020.101852. arXiv: 1906.02076. URL: https://doi.org/ $10.1016 / \mathrm{j}$. artmed.2020.101852.

Chen, He, Yan Song, and Xiaoli Li (2019). "A deep learning framework for identifying children with ADHD using an EEG-based brain network". In: Neurocomputing 356, pp. 83-96. ISSN: 18728286. DOI: 10.1016/j .neucom.2019.04.058. URL: https://doi.org/10.1016/j.neucom.2019.04.058.

Cho, Kyunghyun et al. (2014). "On the properties of neural machine translation: Encoder-decoder approaches". In: arXiv preprint arXiv:1409.1259.

Clarke, Shannon et al. (2019). "Computer-assisted EEG diagnostic review for idiopathic generalized epilepsy". In: Epilepsy \& Behavior, p. 106556. ISSN: 15255050. DOI: https : / / doi .org/10 . 1016/j . yebeh . 2019 . 106556. URL: http: //www.sciencedirect.com/science/article/pii/S1525505019306018.

Craik, Alexander, Yongtian He, and Jose L. Contreras-Vidal (2019). "Deep learning for electroencephalogram (EEG) classification tasks: A review". In: Journal of Neural Engineering 16.3. ISSN: 17412552. DOI: 10.1088/1741-2552/ab0ab5.

Croskerry, Pat (2003). "The importance of cognitive errors in diagnosis and strategies to minimize them". In: Academic Medicine 78.8, pp. 775-780. ISSN: 10402446. DOI: $10.1097 / 00001888-200308000-00003$.

Dash, Dianne et al. (2012). "Ambulatory EEG: A cost-effective alternative to inpatient video-EEG in adult patients". In: Epileptic Disorders 14.3, pp. 290297. ISSN: 12949361. DOI: 10.1684/epd.2012.0529.

Dean, Dennis A. et al. (2016). "Scaling Up Scientific Discovery in Sleep Medicine: The National Sleep Research Resource". In: Sleep 39.5, pp. 1151-1164. IsSN: 0161-8105. DOI: 10.5665/sleep. 5774.

Domingues, Inês et al. (2019). "Using deep learning techniques in medical imaging: a systematic review of applications on CT and PET". In: Artificial Intelligence Review, pp. 1-68. 
Dominic, Anna, K. J. Aswathy, and Surekha Mariam Varghese (2019). "Deep learning in computer aided diagnosis of MDD". In: International Journal of Innovative Technology and Exploring Engineering 8.6, pp. 464-468. ISSN: 22783075.

Dubreuil-Vall, Laura, Giulio Ruffini, and Joan A. Camprodon (2020). "Deep Learning Convolutional Neural Networks Discriminate Adult ADHD From Healthy Individuals on the Basis of Event-Related Spectral EEG". In: Frontiers in Neuroscience 14.April, pp. 1-12. ISSN: 1662453X. DOI: 10.3389/fnins.2020.00251.

Dupuy, F. E. et al. (2014). "EEG and electrodermal activity in girls with attentiondeficit/hyperactivity disorder". In: Clinical Neurophysiology 125.3, pp. 491499. ISSN: 13882457. DOI: $10.1016 / \mathrm{j}$. clinph . 2013 . 09 . 007. URL: http : //dx.doi.org/10.1016/j.clinph.2013.09.007.

Emami, Ali et al. (2019). "Seizure detection by convolutional neural networkbased analysis of scalp electroencephalography plot images". In: NeuroImage: Clinical 22, p. 101684. ISSN: 2213-1582. DOI: https://doi .org/10.1016/j . nicl.2019.101684. URL: http://www. sciencedirect.com/science/article/ $\mathrm{pii} / \mathrm{S} 2213158219300348$.

Faulkner, Howard J, Hisatomi Arima, and Armin Mohamed (2012). "The utility of prolonged outpatient ambulatory EEG". In: Seizure 21.7, pp. 491-495. ISSN: 10591311. DOI: $10.1016 / \mathrm{j}$.seizure.2012.04.015. URL: http://dx.doi.org/ 10.1016/j . seizure.2012.04.015.

Ferri, Cleusa P et al. (2005). "Global prevalence of dementia: A Delphi consensus study". In: Lancet 366.9503, pp. 2112-2117. ISSN: 01406736. DOI: $10.1016 /$ S0140-6736(05) 67889-0.

Fisher, Robert S. et al. (2005). Epileptic seizures and epilepsy: Definitions proposed by the International League Against Epilepsy (ILAE) and the International Bureau for Epilepsy (IBE). DOI: 10.1111/j.0013-9580.2005.66104.x.

Fürbass, Franz et al. (2020). "An artificial intelligence-based EEG algorithm for detection of epileptiform EEG discharges: Validation against the diagnostic gold standard". In: Clinical Neurophysiology 131.6, pp. 1174-1179. ISSN: 18728952. DOI: $10.1016 / \mathrm{j} . \mathrm{clinph} .2020 .02 .032$.

Gallagher, Anne et al. (2008). "Non-invasive pre-surgical investigation of a 10 yearold epileptic boy using simultaneous EEG-NIRS". In: Seizure 17.6, pp. 576582. ISSN: 10591311. DOI: 10.1016/j.seizure.2008.01.009.

Geschwind, Michael D et al. (2008). Rapidly progressive dementia. DOI: 10.1002/ ana. 21430.

Girshick, Ross (2015). "Fast r-cnn". In: Proceedings of the IEEE international conference on computer vision, pp. 1440-1448.

Gorbachevskaya, N. N. and S. Borisov (2002). EEG data of healthy adolescents and adolescents with symptoms of schizophrenia. URL: http://brain. bio. msu.ru/eeg_schizophrenia.htm (visited on 06/10/2020).

Graves, Alex and Jürgen Schmidhuber (2005). "Framewise phoneme classification with bidirectional LSTM and other neural network architectures". In: Neural networks 18.5-6, pp. 602-610.

Halász, Péter et al. (2004). "The nature of arousal in sleep". In: Journal of sleep research 13.1, pp. 1-23.

Hinton, Geoffrey E and Ruslan R Salakhutdinov (2006). "Reducing the dimensionality of data with neural networks". In: science 313.5786 , pp. 504-507.

Hochreiter, Sepp and Jürgen Schmidhuber (1997). "Long short-term memory". In: Neural computation 9.8, pp. 1735-1780. 
Hosseini, Mohammad Parsa, Amin Hosseini, and Kiarash Ahi (2020). "A Review on Machine Learning for EEG Signal Processing in Bioengineering". In: IEEE Reviews in Biomedical Engineering 3333.c. ISSN: 19411189. DOI: 10.1109/RBME. 2020. 2969915.

$\mathrm{Hu}$, Li and Zhiguo Zhang (2019). EEG signal processing and feature extraction. Springer Singapore, pp. 1-437. ISBN: 9789811391132. DOI: 10.1007/978-98113-9113-2.

Hussein, Ramy et al. (2019). "Optimized deep neural network architecture for robust detection of epileptic seizures using EEG signals". In: Clinical Neurophysiology 130.1, pp. 25-37. ISSN: 18728952. DOI: 10.1016/j.clinph.2018.10.010. URL: https://doi.org/10.1016/j.clinph.2018.10.010.

Ieracitano, Cosimo et al. (2020). "A novel multi-modal machine learning based approach for automatic classification of EEG recordings in dementia". In: Neural Networks 123, pp. 176-190. ISSN: 18792782. DOI: 10.1016/j.neunet.2019.12. 006. URL: https://doi.org/10.1016/j.neunet.2019.12.006.

Jankovic, J (2008). Parkinson's disease: Clinical features and diagnosis. DOI: 10 . 1136/jnnp. 2007.131045. URL: http://jnnp.bmj.com/.

Jeste, Shafali S., Joel Frohlich, and Sandra K. Loo (2015). Electrophysiological biomarkers of diagnosis and outcome in neurodevelopmental disorders. DOI: 10 . 1097 / WCO . 0000000000000181 . URL: http : / / content . wkhealth . com / linkback / openurl ? sid = WKPTLP : landingpage \& an =00019052 - 201504000 00005.

Johnson, Chris Plauché et al. (2007). Identification and evaluation of children with autism spectrum disorders. DOI: 10.1542/peds.2007-2361.

Jonas, Stefan et al. (2019). "EEG-based outcome prediction after cardiac arrest with convolutional neural networks: Performance and visualization of discriminative features". In: Human Brain Mapping 40.16, pp. 4606-4617. ISSN: 10970193. DOI: $10.1002 / \mathrm{hbm} .24724$.

Jurcak, Valer, Daisuke Tsuzuki, and Ippeita Dan (2007). "10/20, 10/10, and 10/5 systems revisited: Their validity as relative head-surface-based positioning systems". In: NeuroImage 34.4, pp. 1600-1611. ISSN: 10538119. DOI: 10.1016/j . neuroimage.2006.09.024.

Khan, Haidar et al. (2018). "Focal onset seizure prediction using convolutional networks". In: IEEE Transactions on Biomedical Engineering 65.9, pp. 2109 2118. ISSN: 15582531. DOI: 10.1109/TBME.2017.2785401. arXiv: 1805.11576.

Kieling, Renata and Luis A. Rohde (2012). "ADHD in children and adults: Diagnosis and prognosis". In: Current Topics in Behavioral Neurosciences 9, pp. 116. ISSN: 18663389. DOI: 10.1007/7854_2010_115.

Kingma, Diederik P and Max Welling (2014). "Auto-encoding variational bayes". In: 2nd International Conference on Learning Representations, ICLR 2014 Conference Track Proceedings. arXiv: 1312.6114.

Kitchenham, Barbara and Stuart Charters (2007). "Guidelines for performing systematic literature reviews in software engineering". In:

Kitchenham, Barbara A, David Budgen, and O Pearl Brereton (2011). "Using mapping studies as the basis for further research-a participant-observer case study". In: Information and Software Technology 53.6, pp. 638-651.

LeCun, Yann et al. (1998). "Gradient-based learning applied to document recognition". In: Proceedings of the IEEE 86.11, pp. 2278-2324. 
Li, Xiaowei et al. (2019a). "Depression recognition using machine learning methods with different feature generation strategies". In: Artificial Intelligence in Medicine 99, p. 101696. ISSN: 0933-3657. DOI: https ://doi.org/10.1016/ j . artmed . 2019.07 .004. URL: http : //www . sciencedirect . com/science/ article/pii/S0933365719300296.

Li, Xiaowei et al. (2020a). "A Deep Learning Approach for Mild Depression Recognition Based on Functional Connectivity Using Electroencephalography". In: Frontiers in Neuroscience 14.April, pp. 1-20. ISSN: 1662453X. DOI: 10.3389/ fnins.2020.00192.

Li, Yang et al. (2019b). "Epileptic seizure detection in EEG signals using sparse multiscale radial basis function networks and the Fisher vector approach". In: Knowledge-Based Systems 164, pp. 96-106.

Li, Yang et al. (2020b). "Automatic Seizure Detection using Fully Convolutional Nested LSTM". In: International Journal of Neural Systems 30.4. ISSN: 17936462. DOI: $10.1142 / \mathrm{S} 0129065720500197$.

Liang, Weixia et al. (2019). "Scalp EEG epileptogenic zone recognition and localization based on long-term recurrent convolutional network". In: Neurocomputing. ISSN: 0925-2312. DOI: https : // doi .org/10 . 1016/j . neucom . 2018. 10. 108. URL: http : / / www . sciencedirect . com / science/article / pii / S0925231219304928.

Liu, Guoyang, Weidong Zhou, and Minxing Geng (2019). "Automatic Seizure Detection Based on S-Transform and Deep Convolutional Neural Network". In: International Journal of Neural Systems 1950024, pp. 1-15. ISSN: 17936462. DOI: $10.1142 / \mathrm{S} 0129065719500242$.

Liu, Yuan et al. (2020). "Deep C-LSTM Neural Network for Epileptic Seizure and Tumor Detection Using High-Dimension EEG Signals". In: IEEE Access 8, pp. 37495-37504. ISSN: 21693536. DOI: 10.1109/ACCESS . 2020.2976156.

Lundberg, Scott M and Su-In Lee (2016). SHapley Additive exPlanations. https: //github.com/slundberg/shap.

- (2017). "A Unified Approach to Interpreting Model Predictions". In: Advances in Neural Information Processing Systems 30. Ed. by I. Guyon et al. Curran Associates, Inc., pp. 4765-4774. URL: http://papers.nips.cc/paper/7062a-unified-approach-to-interpreting-model-predictions.pdf.

Masci, Jonathan et al. (2011). "Stacked convolutional auto-encoders for hierarchical feature extraction". In: Lecture Notes in Computer Science (including subseries Lecture Notes in Artificial Intelligence and Lecture Notes in Bioinformatics). Vol. 6791 LNCS. PART 1, pp. 52-59. ISBN: 9783642217340. DOI: 10. 1007 / 978-3-642-21735-7_7. URL: https : / / www . researchgate . net/ publication/221078713.

Mcloughlin, Gráinne, Scott Makeig, and Ming T. Tsuang (2014). "In search of biomarkers in psychiatry: EEG-based measures of brain function". In: American Journal of Medical Genetics, Part B: Neuropsychiatric Genetics 165.2, pp. 111-121. ISSN: 15524841. DOI: 10.1002/ajmg.b.32208.

Mecarelli, Oriano (2019). "Electrode Placement Systems and Montages". In: Clinical Electroencephalography. Ed. by Oriano Mecarelli. Cham: Springer International Publishing, pp. 35-52. ISBN: 978-3-030-04573-9. DOI: 10.1007/978-3030-04573-9_4. URL: https://doi.org/10.1007/978-3-030-04573-9_4.

Merlin Praveena, D., D. Angelin Sarah, and S. Thomas George (2020). "Deep Learning Techniques for EEG Signal Applications-a Review". In: IETE Jour- 
nal of Research 2063. ISSN: 0974780X. DOI: 10.1080/03772063.2020.1749143. URL: https://doi.org/10.1080/03772063.2020.1749143.

Miotto, Riccardo et al. (2017). "Deep learning for healthcare: Review, opportunities and challenges". In: Briefings in Bioinformatics 19.6, pp. 1236-1246. ISSN: 14774054. DOI: $10.1093 / \mathrm{bib} / \mathrm{bbx} 044$.

Morabito, Francesco Carlo et al. (2017). "Deep learning representation from electroencephalography of early-stage creutzfeldt-jakob disease and features for differentiation from rapidly progressive dementia". In: International Journal of Neural Systems 27.2, pp. 1-15. ISSN: 01290657. DOI: 10.1142/S0129065716500398.

Mumtaz, Wajid and Abdul Qayyum (2019). "A deep learning framework for automatic diagnosis of unipolar depression". In: International Journal of Medical Informatics 132.June, p. 103983. ISSN: 18728243. DOI: 10.1016/j.ijmedinf . 2019.103983. URL: https://doi .org/10.1016/j . ijmedinf .2019.103983.

Murtaza, Ghulam et al. (2019). "Deep learning-based breast cancer classification through medical imaging modalities: state of the art and research challenges". In: Artificial Intelligence Review, pp. 1-66.

Naira, Carlos Alberto Torres and Cristian José López Del Alamo (2019). "Classification of people who suffer schizophrenia and healthy people by EEG signals using deep learning". In: International Journal of Advanced Computer Science and Applications 10.10, pp. 511-516. ISSN: 21565570. DOI: 10.14569/ijacsa. 2019.0101067. URL: www.ijacsa.thesai.org.

Noor, Nor Safira Elaina Mohd and Haidi Ibrahim (2020). "Machine Learning Algorithms and Quantitative Electroencephalography Predictors for Outcome Prediction in Traumatic Brain Injury: A Systematic Review". In: IEEE Access 8, pp. 102075-102092. ISSN: 21693536. DOI: 10.1109/ACCESS. 2020. 2998934.

Obeid, Iyad and Joseph Picone (2016). "The temple university hospital EEG data corpus". In: Frontiers in Neuroscience 10.MAY, p. 196. ISSN: 1662453X. DOI: 10 . 3389 / fnins . 2016 . 00196. URL: http : / / journal . frontiersin . org / Article/10.3389/fnins.2016.00196/abstract.

Oh, Shu Lih et al. (2019). "Deep Convolutional Neural Network Model for Automated Diagnosis of Schizophrenia Using EEG Signals". In: Applied Sciences 9.14, p. 2870. ISSN: 2076-3417. DOI: 10.3390/app9142870.

Olbrich, Sebastian and Martijn Arns (2013). "EEG biomarkers in major depressive disorder: Discriminative power and prediction of treatment response". In: International Review of Psychiatry 25.5, pp. 604-618. ISSN: 09540261. DOI: 10.3109/09540261.2013.816269. URL: https://www . tandfonline.com/doi/ abs/10.3109/09540261.2013.816269.

Olbrich, Sebastian, Rik Van Dinteren, and Martijn Arns (2016). Personalized Medicine: Review and Perspectives of Promising Baseline EEG Biomarkers in Major Depressive Disorder and Attention Deficit Hyperactivity Disorder. DOI: 10.1159/000437435. URL: https://www.karger.com/Article/FullText/ 437435https: //www.karger. com/Article/Abstract/437435.

Organization, World Health (2001). The World Health Report 2001: Mental health: new understanding, new hope. World Health Organization.

Petersen, Kai et al. (2008). "Systematic mapping studies in software engineering". In: 12th International Conference on Evaluation and Assessment in Software Engineering (EASE) 12, pp. 1-10.

Phang, Chun Ren et al. (2020). "A Multi-Domain Connectome Convolutional Neural Network for Identifying Schizophrenia From EEG Connectivity Patterns". 
In: IEEE journal of biomedical and health informatics 24.5, pp. 1333-1343. ISSN: 21682208. DOI: 10.1109/JBHI .2019.2941222.

Picchietti, Daniel and John W Winkelman (2005). "Restless legs syndrome, periodic limb movements in sleep, and depression". In: Sleep 28.7, pp. 891-898.

Podgorelec, V. (2012). "Analyzing EEG signals with machine learning for diagnosing alzheimer's disease". In: Elektronika ir Elektrotechnika 18.8, pp. 61-64. ISSN: 13921215. DOI: 10.5755/j01.eee.18.8.2627.

Poirson, Patrick and Haroon Idrees (2013). "Multimodal Stacked Denoising Autoencoders". In: Technical Report, pp. 1-4.

Roth, Thomas (2007). "Insomnia: definition, prevalence, etiology, and consequences". In: Journal of clinical sleep medicine 3.5 suppl, S7-S10.

Roy, Yannick et al. (2019). "Deep learning-based electroencephalography analysis: A systematic review". In: Journal of Neural Engineering 16.5. ISSN: 17412552. DOI: $10.1088 / 1741-2552 / a b 260$ c. arXiv: 1901.05498.

Ruffini, Giulio et al. (2019). "Deep learning with EEG spectrograms in rapid eye movement behavior disorder". In: Frontiers in Neurology 10.JUL, pp. 1-9. ISSN: 16642295. DOI: 10.3389/fneur.2019.00806.

Rumelhart, David E, Geoffrey E Hinton, and Ronald J Williams (1986). "Learning representations by back-propagating errors". In: nature 323.6088 , pp. 533-536.

Sahu, Rekha et al. (2020). "Epileptic seizure detection: a comparative study between deep and traditional machine learning techniques". In: Journal of integrative neuroscience 19.1, pp. 1-9. ISSN: 02196352. DOI: $10.31083 / \mathrm{j} \cdot \mathrm{j}$ in . 2020.01 .24 .

Shahin, Mostafa et al. (2017). "Deep Learning and Insomnia: Assisting Clinicians with Their Diagnosis". In: IEEE Journal of Biomedical and Health Informatics 21.6, pp. 1546-1553. ISSN: 21682194. DOI: 10.1109/JBHI.2017.2650199.

Sharma, Rishi Raj and Ram Bilas Pachori (2017). "Time-frequency representation using IEVDHM-HT with application to classification of epileptic EEG signals". In: IET Science, Measurement \& Technology 12.1, pp. 72-82.

Sharma, Rishi Raj et al. (2018). "Automated system for epileptic EEG detection using iterative filtering". In: IEEE Sensors Letters 2.4, pp. 1-4.

Shoeb, Ali (2009). "Application of machine learning to epileptic seizure onset detection and treatment". In: Diss. Massachusetts Institute of Technology, pp. 157-162. URL: https : / / dspace . mit . edu/handle/1721 .1/54669http: //dspace.mit.edu/handle/1721.1/54669.

Shrestha, Ajay and Ausif Mahmood (2019). "Review of deep learning algorithms and architectures". In: IEEE Access 7, pp. 53040-53065.

Smolensky, P (1986). "Information processing in dynamical systems: Foundations of harmony theory BT - Parallel Distributed Processing: Volume 1: Foundations". In: Parallel Distributed Processing: Volume 1: Foundations. Cambridge, MA, USA: MIT Press, pp. 194-281. ISBN: 026268053X.

Sun, Mingrui et al. (2018). "Prediction for high risk clinical symptoms of epilepsy based on deep learning algorithm". In: IEEE Access 6, pp. 77596-77605. ISSN: 21693536. DOI: 10.1109/ACCESS. 2018.2883562.

Sun, Shiliang and Jin Zhou (2014). "A review of adaptive feature extraction and classification methods for EEG-based brain-computer interfaces". In: Proceedings of the International Joint Conference on Neural Networks, pp. 1746-1753. DOI: 10.1109/IJCNN . 2014.6889525. 
Thara, T. D.K., B. G.P. PremaSudha, and Fan Xiong (2019). "Epileptic seizure detection and prediction using stacked bidirectional long short term memory". In: Pattern Recognition Letters 128, pp. 529-535. ISSN: 01678655. DOI: 10 . 1016/j ·patrec . 2019 . 10.034. URL: https : //doi .org/10 . 1016/j · patrec . 2019.10.034.

Tjepkema-Cloostermans, Marleen C. et al. (2019). "Outcome Prediction in Postanoxic Coma With Deep Learning". In: Critical care medicine 47.10, pp. 1424-1432. ISSN: 15300293 . DOI: 10.1097/CCM. 0000000000003854.

Tsiouris, ?ostas et al. (2018). "A Long Short-Term Memory deep learning network for the prediction of epileptic seizures using EEG signals". In: Computers in Biology and Medicine 99.March, pp. 24-37. ISSN: 18790534. DOI: 10.1016/j . compbiomed.2018.05.019.

Türk, Ömer and Mehmet Siraç Özerdem (2019). "Epilepsy detection by using scalogram based convolutional neural network from eeg signals". In: Brain Sciences 9.5. ISSN: 20763425. DOI: 10.3390/brainsci9050115.

University of Freiburg (2003). EEG Database - Seizure Prediction Project Freiburg. URL: http : //epilepsy - uni-freiburg . de/freiburg-seizure-predictionproject/ eeg-databasehttps : / / epilepsy . uni-freiburg . de / freiburgseizure-prediction-project/eeg-database.

Vahid, Amirali et al. (2019). "Deep Learning Based on Event-Related EEG Differentiates Children with ADHD from Healthy Controls". In: Journal of Clinical Medicine 8.7, p. 1055. ISSN: 2077-0383. DOI: $10.3390 / \mathrm{jcm} 8071055$.

Vincent, Pascal et al. (2010). "Stacked denoising autoencoders: Learning useful representations in a deep network with a local denoising criterion." In: Journal of machine learning research 11.12 .

Warrick, Philip A., Vincent Lostanlen, and Masun Nabhan Homsi (2019). "Hybrid scattering-LSTM networks for automated detection of sleep arousals". In: Physiological Measurement 40.7. ISSN: 13616579. DOI: 10.1088/1361-6579/ ab2664.

Wei, Xiaoyan et al. (2018). "Automatic seizure detection using three-dimensional CNN based on multi-channel EEG". In: BMC Medical Informatics and Decision Making 18.Suppl 5. ISSN: 14726947. DOI: 10.1186/s12911-018-0693-8.

Wei, Zuochen et al. (2019). "Automatic epileptic EEG detection using convolutional neural network with improvements in time-domain". In: Biomedical Signal Processing and Control 53, p. 101551. ISSN: 17468108. DOI: 10.1016/j . bspc. 2019.04.028. URL: https://doi.org/10.1016/j.bspc.2019.04.028.

Weisman, David and Ian McKeith (2007). "Dementia with Lewy bodies". In: Seminars in Neurology 27.1, pp. 42-47. ISSN: 02718235. DOI: $10.1055 / \mathrm{s}-2006-$ 956754. URL: http://bjp.rcpsych.org/subscriptions/.

Wen, Tingxi and Zhongnan Zhang (2018). "Deep Convolution Neural Network and Autoencoders-Based Unsupervised Feature Learning of EEG Signals". In: IEEE Access 6, pp. 25399-25410. ISSN: 21693536. DOI: 10.1109/ACCESS . 2018. 2833746.

Wittchen, H. U. et al. (2011). "The size and burden of mental disorders and other disorders of the brain in Europe 2010". In: European Neuropsychopharmacology 21.9, pp. 655-679. ISSN: 0924977X. DOI: 10.1016/j .euroneuro.2011.07.018. URL: http://dx.doi.org/10.1016/j. euroneuro.2011.07.018. 
Xie, Ning et al. (2020). Explainable Deep Learning:A Field Guide for the Uninitiated Explainable Deep Learning: A Field Guide for the Uninitiated. Tech. rep. arXiv: $2004.14545 \mathrm{v} 1$.

Yosinski, Jason et al. (2014). "How transferable are features in deep neural networks?" In: Advances in neural information processing systems, pp. 3320-3328.

Young, Terry, Paul E Peppard, and Daniel J Gottlieb (2002). "Epidemiology of obstructive sleep apnea: a population health perspective". In: American journal of respiratory and critical care medicine 165.9, pp. 1217-1239.

Yuan, Ye et al. (2019). "A multi-view deep learning framework for EEG seizure detection". In: IEEE Journal of Biomedical and Health Informatics 23.1, pp. 8394. ISSN: 21682194. DOI: 10.1109/JBHI.2018.2871678.

Zerafa, Rosanne et al. (2018). "A comparison of a broad range of EEG acquisition devices-is there any difference for SSVEP BCIs?" In: Brain-Computer Interfaces 5.4, pp. 121-131. ISSN: 23262621. DOI: 10.1080/2326263X . 2018.1550710. URL: https://doi.org/10.1080/2326263X .2018.1550710.

Zerr, Inga et al. (2009). "Updated clinical diagnostic criteria for sporadic CreutzfeldtJakob disease". In: Brain 132.10, pp. 2659-2668. ISSN: 00068950. DOI: 10 . 1093 / brain / awp191. URL: https : / / academic . oup . com / brain / articleabstract/132/10/2659/331080.

Zhang, Tao and Wanzhong Chen (2016). "LMD based features for the automatic seizure detection of EEG signals using SVM". In: IEEE Transactions on Neural Systems and Rehabilitation Engineering 25.8, pp. 1100-1108.

Zhou, Chunting et al. (2015). "A C-LSTM neural network for text classification". In: arXiv preprint arXiv:1511.08630.

Zhu, Jing et al. (2019). "Multimodal mild depression recognition based on EEGEM synchronization acquisition network". In: IEEE Access 7, pp. 28196-28210. ISSN: 21693536. DOI: 10.1109/ACCESS. 2019.2901950. 
A List of acronyms

Table 7: List of acronyms

Text

Attention Deficit Hyperactivity Disorder

Autoencoder

Artificial Intelligence

Convolutional Long-Short Term Memory

Convolutional Autoencoder

Convolutional Neural Network

Convolutional Variational Autoencoder

Denoising Autoencoder

Deep Learning

Deep Neural Network

Electrocardiogram

Electrodermal Activity

Electroencephalogram

Exponential Linear Unit

Eye Movement

Electromyography

Electrooculography

Feature Engineering

Fast Region-based Convolutional Network

Gated Recurrent Unit

Gradient-Weighted Class Activation Mapping

Long-Short Term Memory

Multimodal Denoising Autoencoder

Multilayer Perceptron

Magnetic Resonance Imaging

Neural Network

Positron Emission Tomography

Restricted Boltzmann Machines

Recurrent Convolutional Neural Network

Recurrent Neural Network

Area Under the Receiver Operator Characteristic

Research Question

Rectified Linear Unit

Stochastic Gradient Descent

Systematic Mapping Study

Siamese Neural Network

Oxygen Saturation

Hyperbolic Tangent

Wasserstein Generative Adversarial Network
Acronym

ADHD

AE

AI

C-LSTM

CAE

CNN

Convolutional VAE

DAE

DI

DNN

ECG

EDA

EEG

ELU

EM

EMG

EOG

FE

Fast R-CNN

GRU

Grad-CAM

LSTM

MDAE

MLP

MRI

NN

PET

RBM

$\mathrm{RCNN}$

RNN

ROC AUC

RQ

ReLU

SGD

SMS

SNN

$\mathrm{SaO} 2$

Tanh

WGAN

\section{B Information about extracted papers}




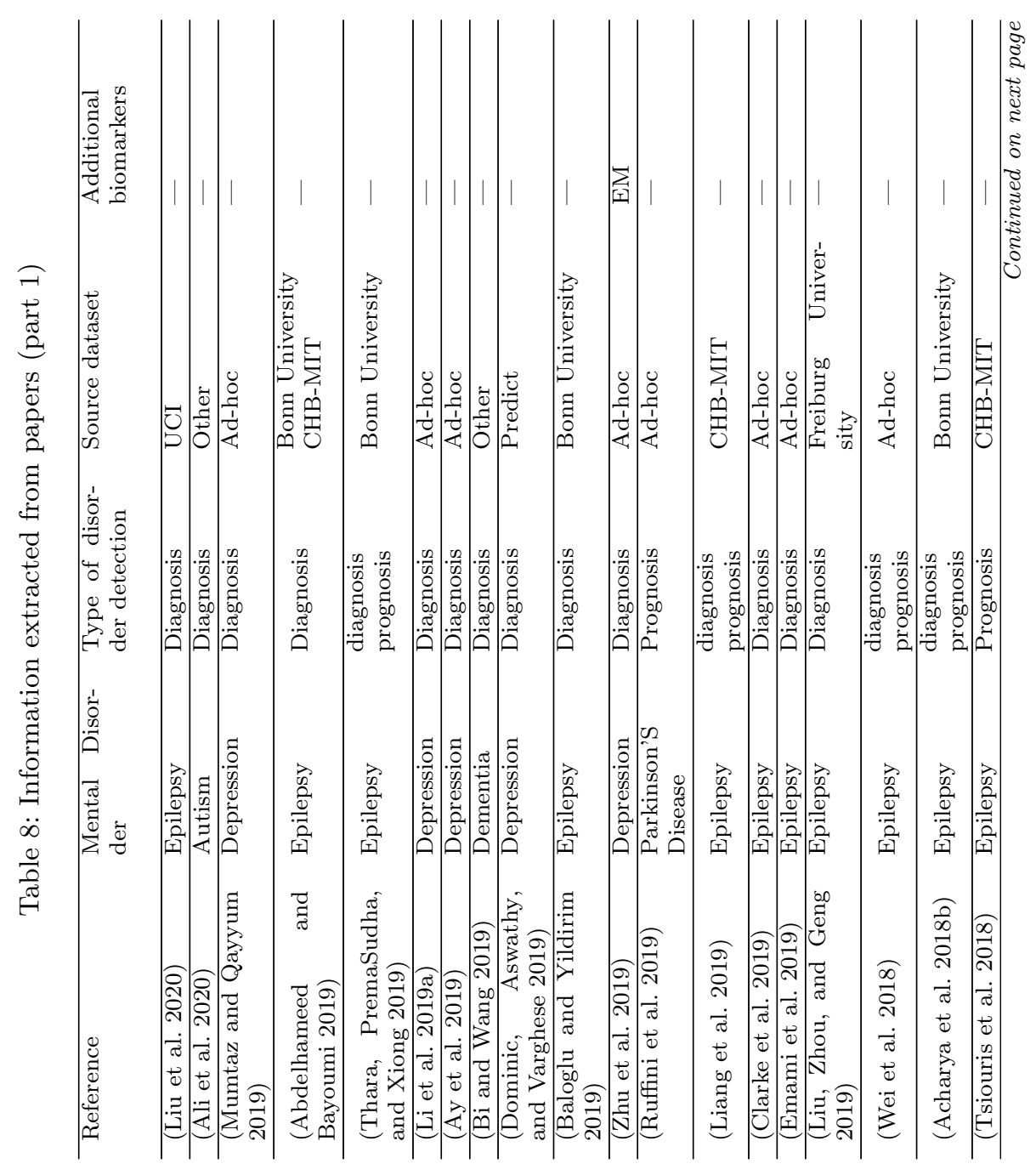




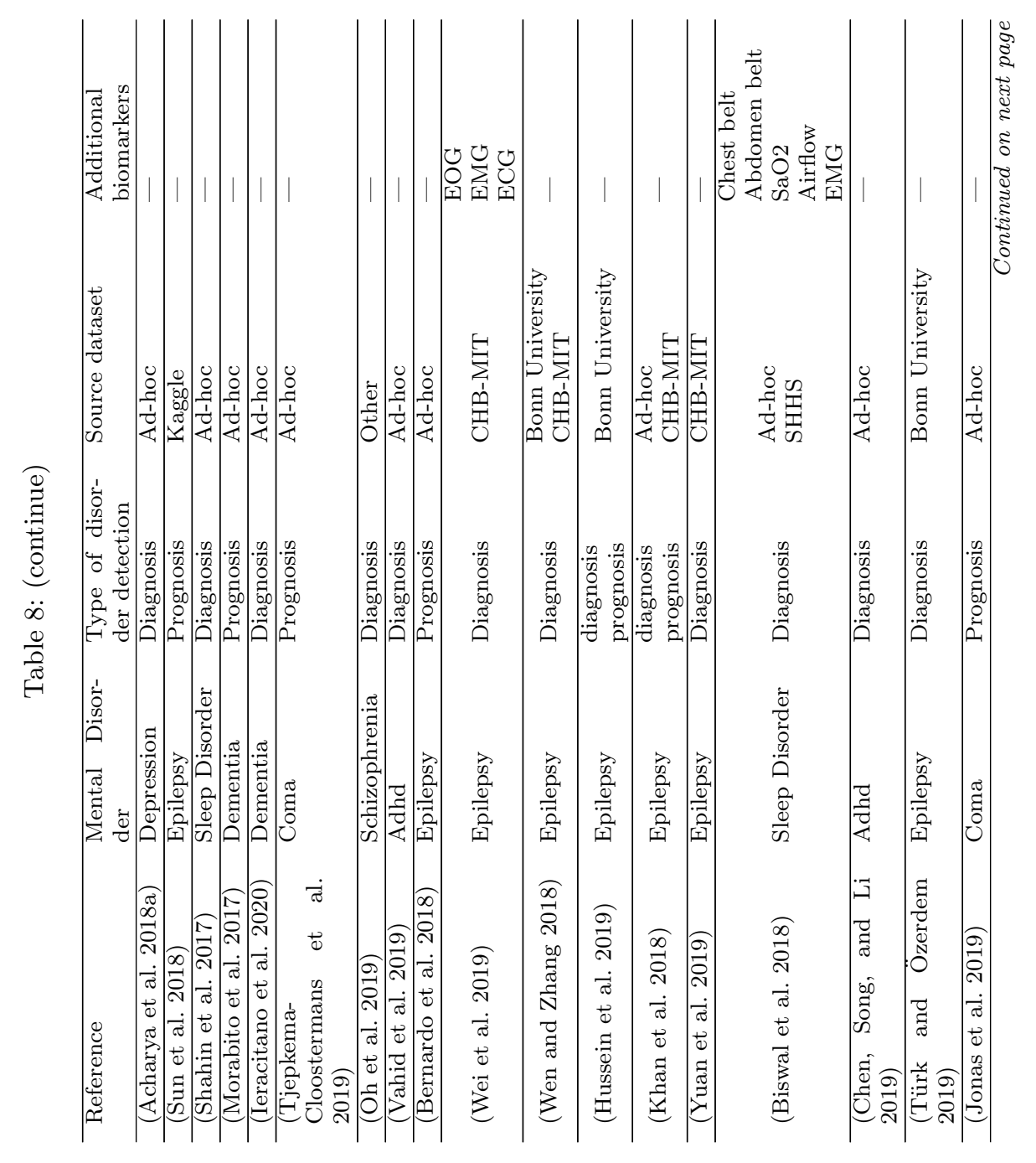




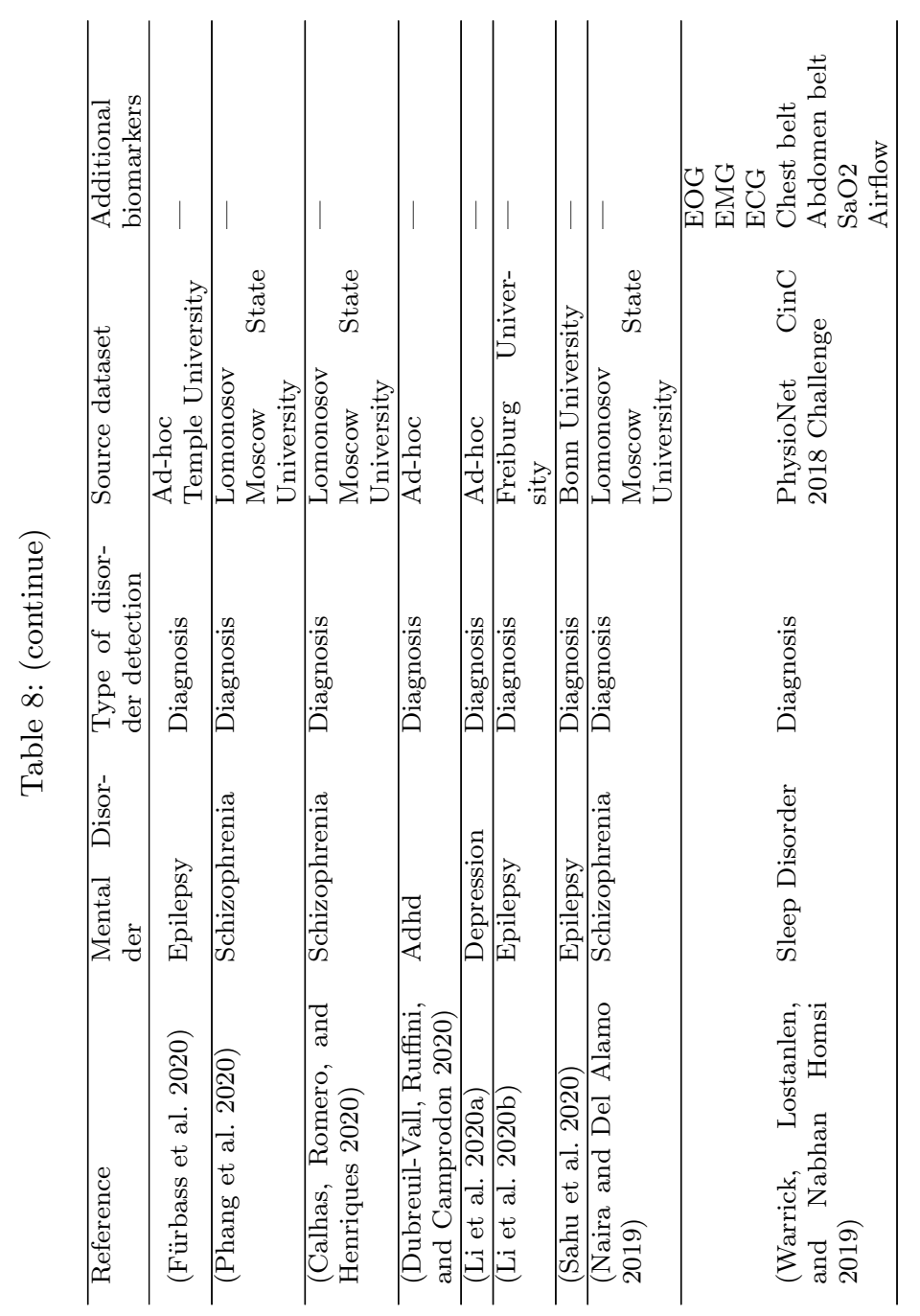




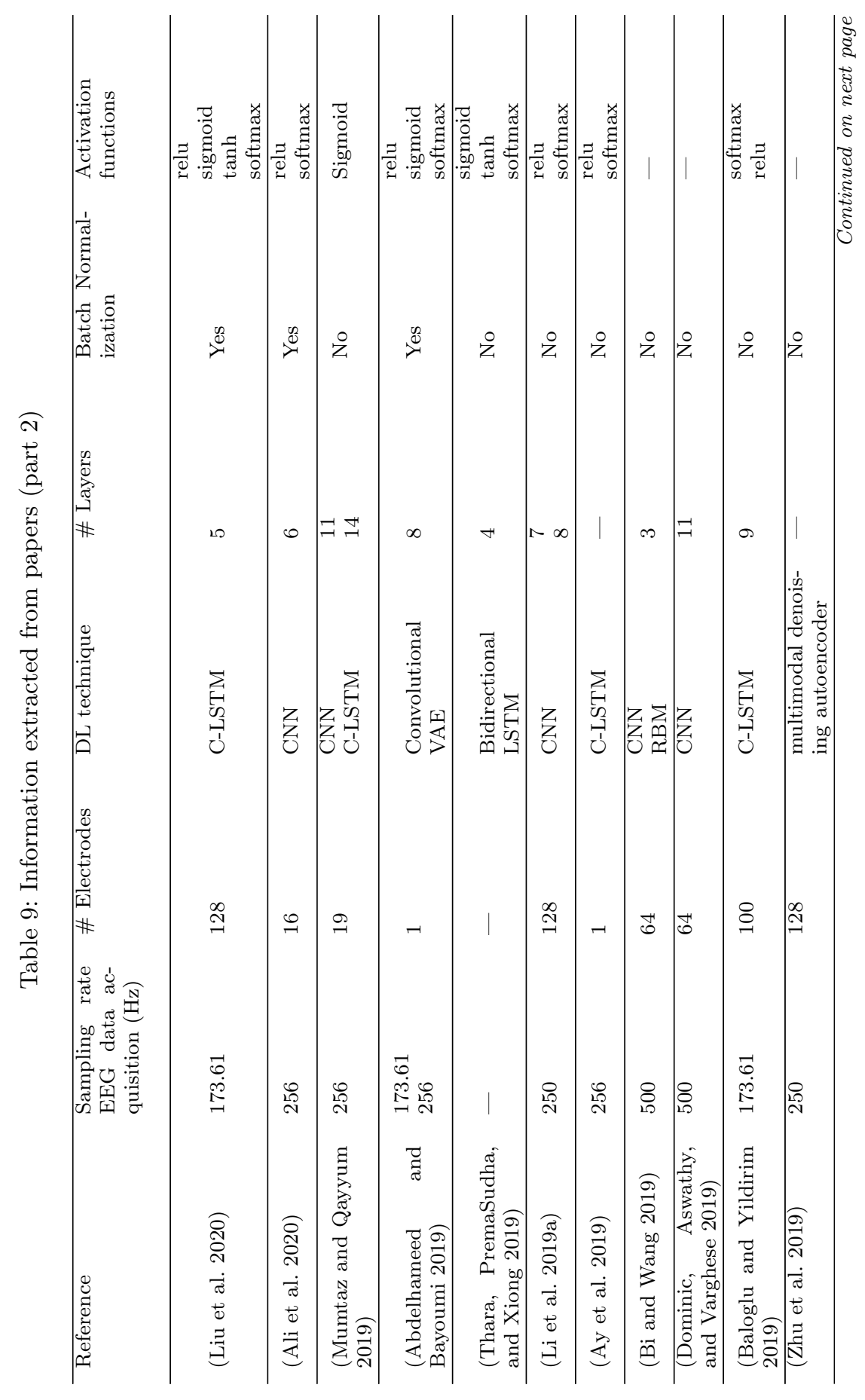




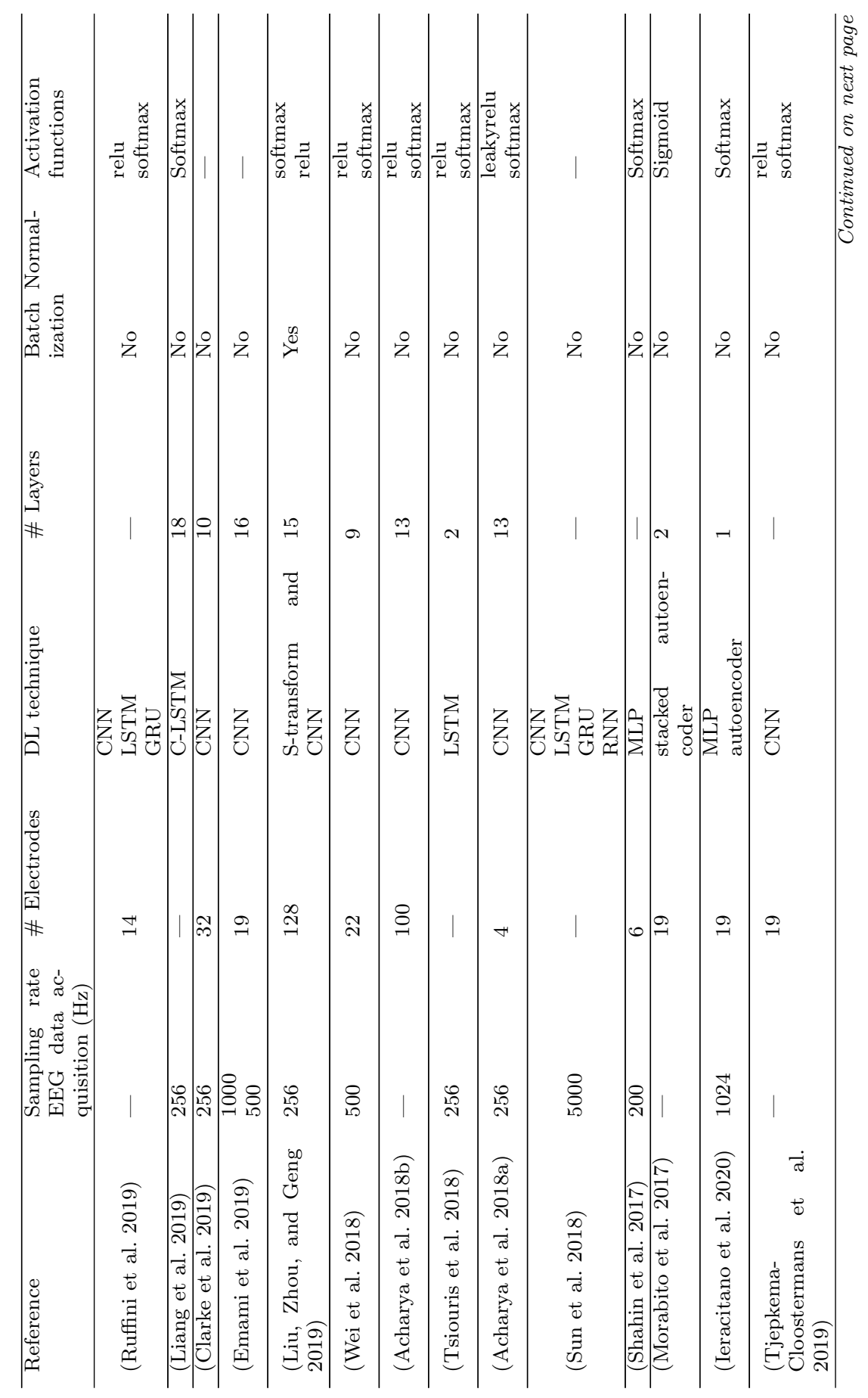




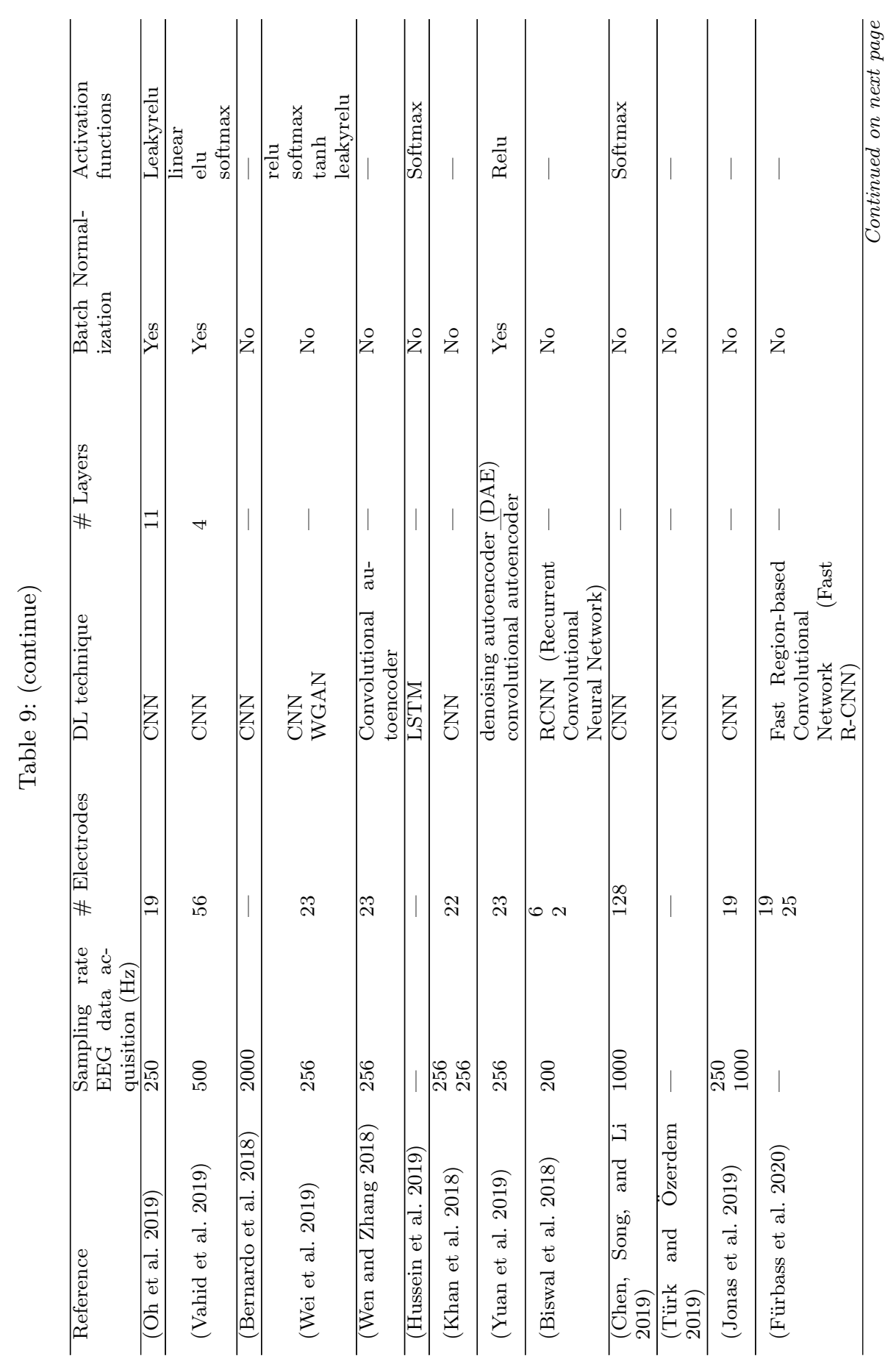




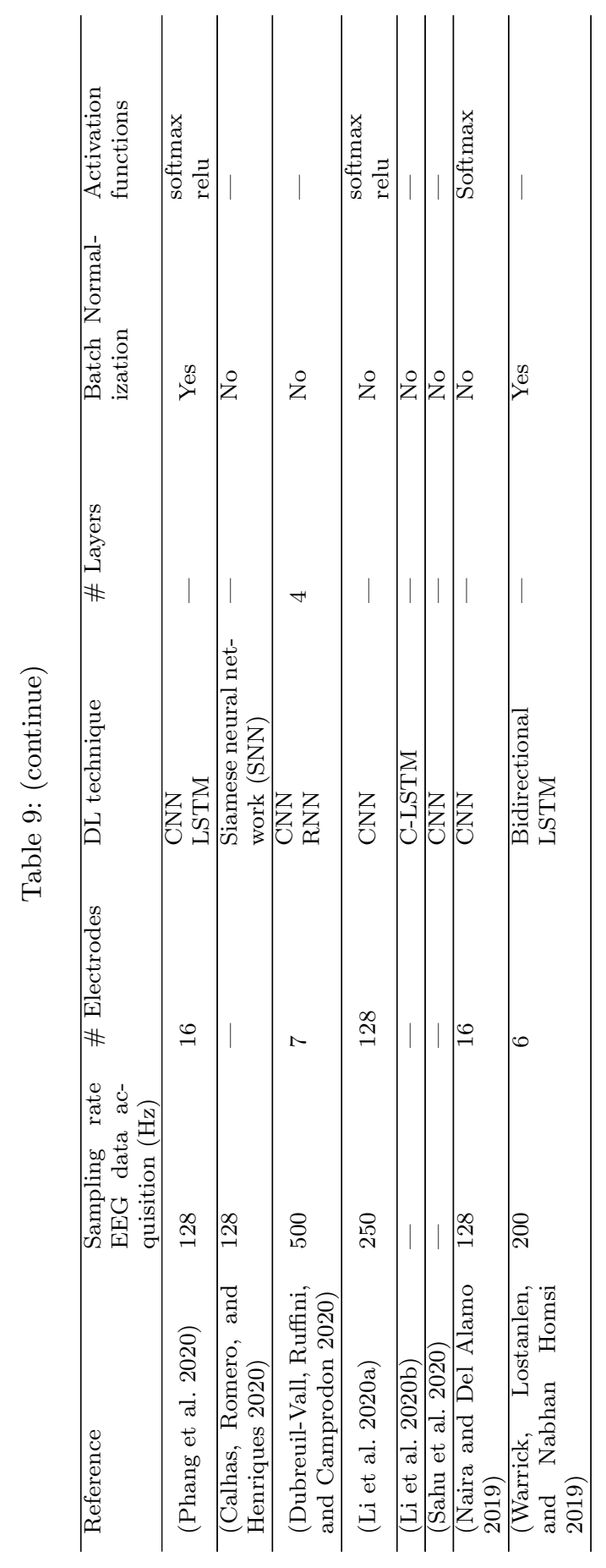




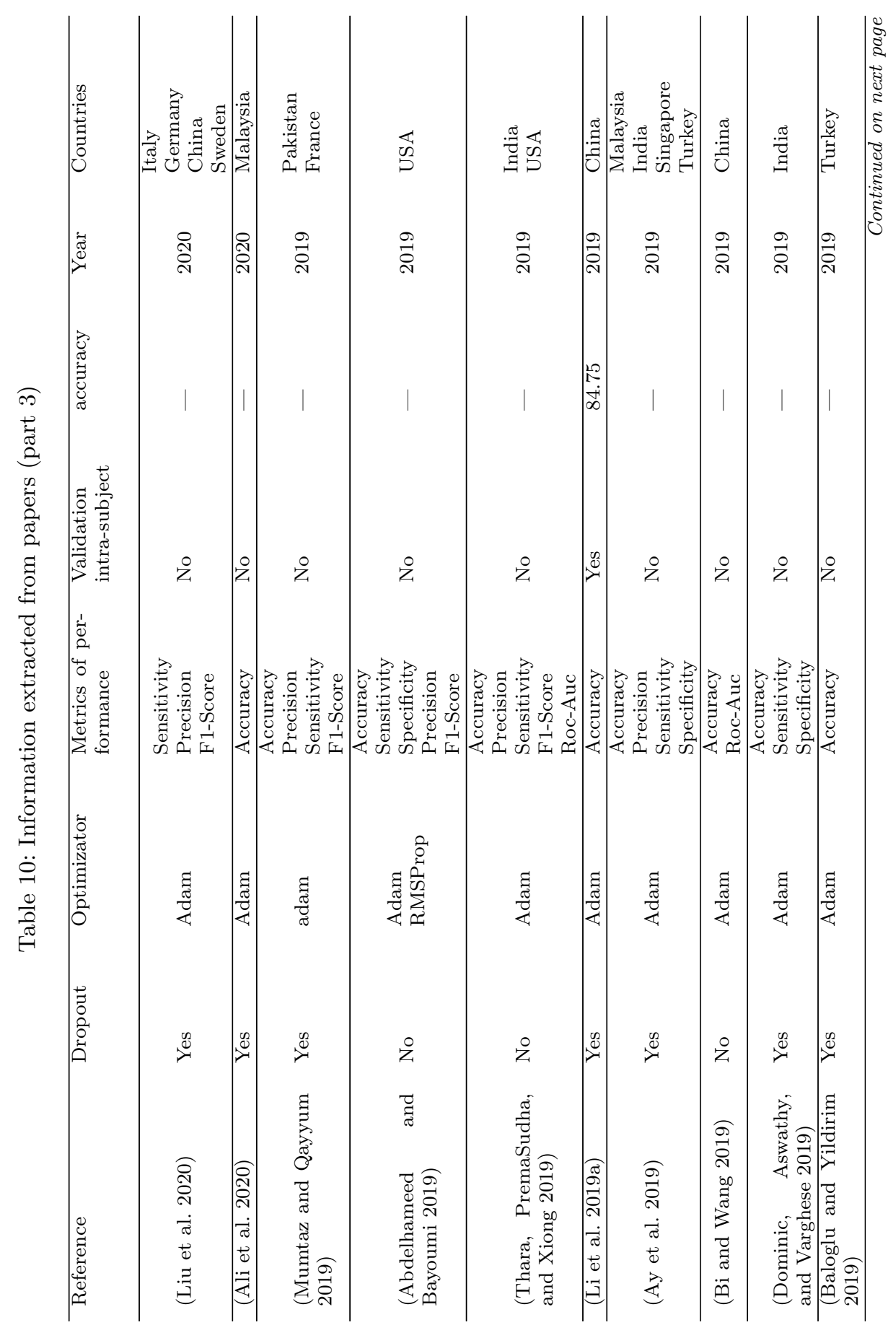




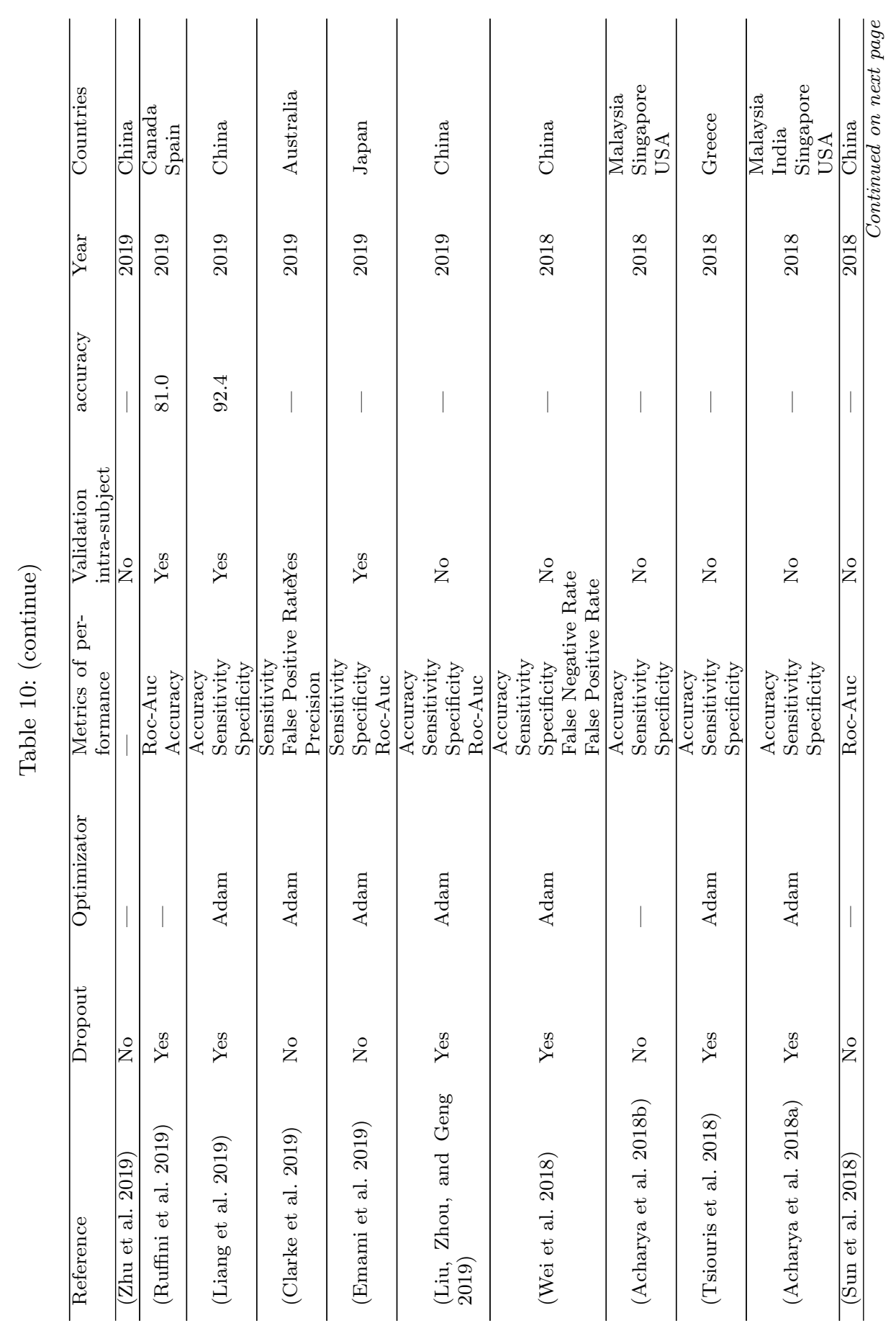




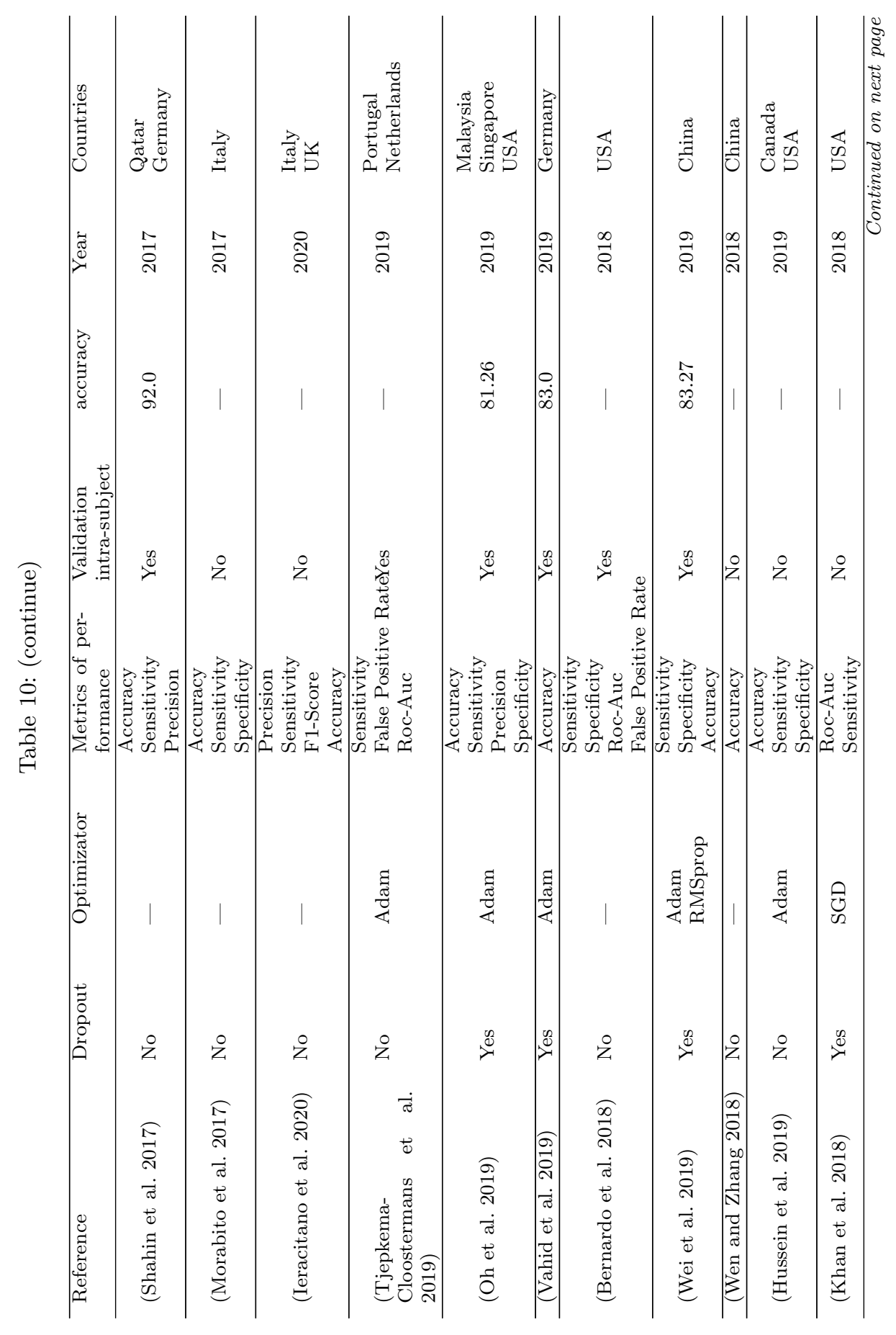




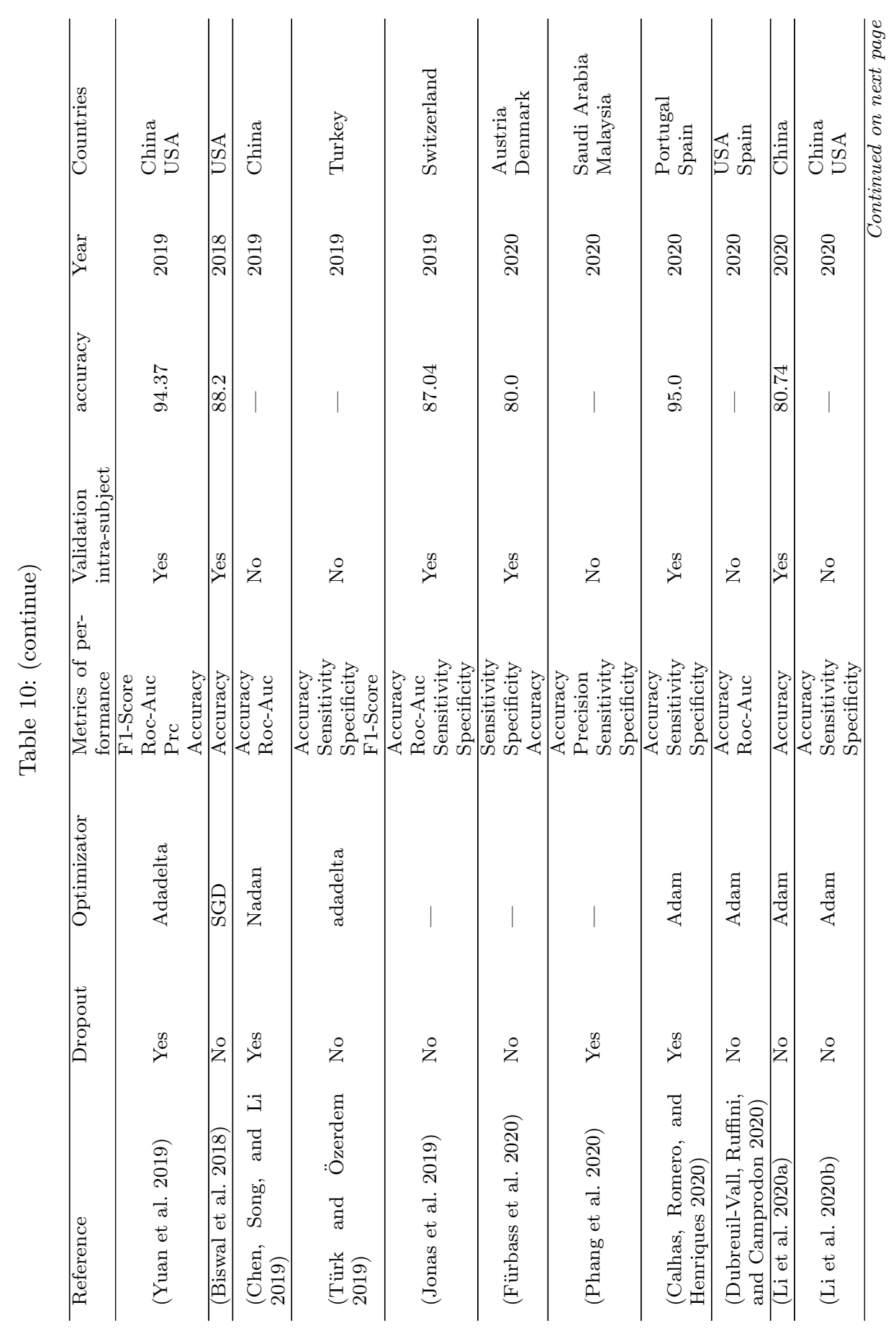




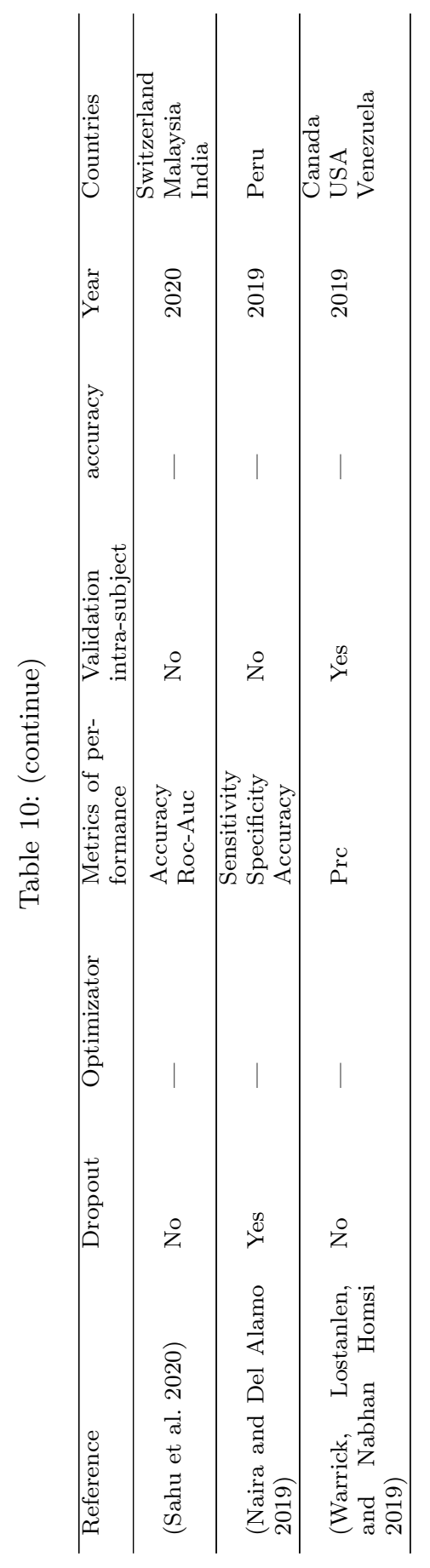

